\title{
Inhibition of Kallikrein-related peptidases 7 restrains pancreatic tumor growth through induction of ferroptosis
}

Yueqing Wang, \#, Fengyi Xiang ${ }^{1,}$, Hao Deng ${ }^{1,}$, Shuang Leng ${ }^{1}$, Dengze Zhao ${ }^{1}$, Yue Chen ${ }^{1,2}$, Donghui Xie ${ }^{1}$, Qingyun Li $^{1}$, Chunyu Cao ${ }^{1}$, Weihong Zheng ${ }^{1}$, Wei Liu ${ }^{2}$, Xiaowen Liu ${ }^{1, *}$, Jun Zheng ${ }^{1,2, *}$, Xiao $\operatorname{Tan}^{1, *}$

${ }^{1}$ Hubei Key Laboratory of Tumor Microenvironment and Immunotherapy, Medical College, China Three Gorges University, Yichang, 443003, P.R. China

${ }^{2}$ The First College of Clinical Medical Science, China Three Gorges University, Yichang, 443003, P.R. China

${ }^{\#}$ These authors contributed equally to this work.

*Authors for correspondence (Xiao Tan, xiao-tan@ctgu.edu.cn; Jun Zheng, zhengjun1995@163.com; Xiaowen Liu, lxw@ctgu.edu.cn). 


\section{Abstract}

Pancreatic cancer is one of the most aggressive and lethal malignancies with extremely poor prognosis, and KLK7 was considered as a potential therapeutic target. In this study, we analyzed the expression of KLK7 in TCGA and GTEx databases and found that $K L K 7$ had a negative correlation to long-term survival rate ( $>1.5$ years) of pancreatic cancer patients. Compound 42 is a coumarinic derivative, a suicide substrate inhibitor of KLK7, which has been proved to inhibit the proliferation of PANC-1 cells in vitro effectively in our previous study. In this study, we further investigated the inhibition ability of Compound 42 in tumor formation and development in CDX and PDX tumor models of pancreatic cancer subsequently. Besides, we studied the inhibitory mechanism of Compound 42 and the result showed that Compound 42 arrested the pancreatic cancer cell cycle in $G_{0} / G_{1}$ phase and induced ferroptosis through down-regulation of GPX4 protein level and accumulation of iron ion. Thus, these experiments demonstrate that Compound 42, suppressing pancreatic cancer in vivo, is expected to become a novel drug for pancreatic cancer treatment.

\section{Key Words}

Kallikrein-related peptidase 7, Pancreatic cancer, Coumarinic derivative, Ferroptosis, Cell cycle arrested 


\section{Introduction}

Pancreatic cancer (PC) is one of the most aggressive and lethal malignancies with extremely poor prognosis, the most common pathological type of $\mathrm{PC}$ is pancreatic ductal adenocarcinoma (PDAC)[1], with concealed symptom, aggressive nature, difficult for early detection, low resection rate, and high recurrence rate after surgery. Currently, only a few PDAC patients are suitable for traditional surgical resection, and the 5-year survival rate of patients after surgery is only about $20 \%$ [2]. Kallikrein-related peptidases 7 (KLK7) is a secreted protein with high expression in PC, which is closely related to the malignant biological behavior of PC[3]. The expression level of KLK7 in PDAC is higher than that in adjacent normal tissues and it is an independent risk factor for vascular invasion of PC[4]. KLK7 can promote the proliferation of pancreatic cancer cells, reduce the chemosensitivity and increase the migration and invasion ability of pancreatic cancer cells[4], which has been demonstrated in our previous in vitro studies[5]. Therefore, the role of KLK7 is critical for PC, targeting KLK7 will provide us a novel strategy for PC treatment.

The dysmetabolism of lipid ROS and the oxidative damage of the cell membrane leads to the destruction of the redox balance in cells[6,7], and are important for ferroptosis which is a novel programmed cell death. Previous studies have found that the highly activated intracellular antioxidant system ensures the survival and adaptability of most pancreatic cancer in the high oxidative tumor microenvironment[8]. Therefore, affecting the antioxidant capacity of pancreatic cancer cells, breaking its steady state of the redox environment and inducing 
ferroptosis can inhibit pancreatic tumor[9]. This strategy has become a hot spot in anti-cancer research and provides us a novel method for treatment of PC.

Coumarin is a heterocyclic compound with $2 \mathrm{H}$-1-benzopyran-2-one structure which has low cytotoxicity and plays an important role in anti-tumor, anti-inflammatory and anti-bacteria research fields[10]. Coumarin derivatives can exert diverse anti-proliferative effects, and some of them such as Irosustat are under clinical trials for the treatment of stomach cancer treatment[11], gastric cancer treatment[12], revealing their potency as putative anticancer drugs[13, 14]. In the previous studies, a series of coumarinic molecules specifically targeting KLK7 was designed and synthesized, the further experiments showed that Compound 42 is a KLK7 suicide inhibitor and can inhibit activity of KLK7 efficiently[15, 16]. Meanwhile, we also found that Compound 42 could effectively inhibit the proliferation, migration and invasion ability of pancreatic cancer cell line PANC-1 in vitro[5], but the inhibitory mechanism was unknown.

Here, we studied inhibitory effect of Compound 42 on CDX and PDX mouse model of PC in vivo and the mechanism of action of Compound 42 through inhibiting KLK7 in pancreatic cancer cells. 


\section{Results}

\section{KLK7 was high expressed in PDAC and negatively correlated with long-term} survival rate of pancreatic cancer patients

In our previous study, expression of KLK7 has been observed up-regulated in 49 PDAC patients[5]. We further discovered that $K L K 7$ was selectively and significantly high expressed in pancreatic cancer, ovarian cancer and thyroid cancer using Cancer Genome Atlas (TCGA) database and Genotype-Tissue Expression (GTEx) database (Fig. 1A). In pancreatic cancer, expression of $K L K 7$ mRNA increased obviously in 178 cancer tissues compared to 171 normal pancreas tissues. Furthermore, we analyzed the relationship between expression of $K L K 7$ mRNA and survival rate of pancreatic cancer patients, the result showed that expression of $K L K 7 \mathrm{mRNA}$ had no impact to short-term survival $(<1.5$ years $)$ rate of pancreatic cancer patients, but the long-term survival (>1.5 years) rate improved significantly when expression of $K L K 7$ mRNA decreased (Fig. 1B).

These results suggest that expression of KLK7 is a biomarker of prognosis of pancreatic cancer, it plays an important role in pancreatic cancer and associates with the malignancy.

\section{Compound 42 suppressed cell vitality in pancreatic cancer cells}

In our previous study, we have demonstrated that silencing $K L K 7$ expression can inhibit proliferation ability of PANC-1 cell in vitro[5]. Previous studies have also found that $K L K 7$ is high expressed in pancreatic cancer cell line BxPC-3[17]. TCGA database showed that there was no KLK7 expression in liver cancer cells (Fig. 1A), 
which was consistent with our previous finding (Sup Fig. 1). Therefore, PANC-1 and BxPC-3 cell lines were considered as the $K L K 7$ positive expression groups, the hepatocarcinoma cell line HepG-2 as the $K L K 7$ negative expression control group in this study.

During a 48-hour treatment with Compound 42 (Structure can be seen in Sup Fig. 4), we observed that the PANC-1 and BxPC-3 cells exhibited morphological changes and the proliferation rate was inhibited significantly when increasing Compound 42 concentration. But under the same treatment condition, the morphology change and proliferation rate of HepG-2 cells remained untouched (Fig. 1C). We further evaluated the cell viability by CCK- 8 experiment and found that the viability of PANC-1 and BxPC-3 cells were significantly decreased in a dose-dependent manner after $48 \mathrm{~h}$ treatment of Compound 42, but it had no obvious effects on the viability of HepG-2 cells (Fig. 1D-F). These results indicate that the inhibition of Compound 42 to PANC-1 and BxPC-3 cell is specific via KLK7 expression.

Compound 42 inhibited the tumor formation and growth rate in cell derived xenograft mouse model and pancreatic cancer patient derived xenograft mouse model

A 39-day toxicity test was performed to determine the toxicity of Compound 42 before treatment assay in vivo, the result showed that Compound 42 had no obvious toxicity to mouse when the treatment concentration of Compound 42 was under 85.0 $\mu \mathrm{g} / \mathrm{g}$ (Sup Fig. 2). Then, we examined the in vivo treatment effect of Compound 42 in 
PANC-1 cell derived xenograft (CDX) mouse model and patient derived xenograft (PDX) mouse model.

CDX mouse model was established by subcutaneous inoculation of PANC-1 cell into SCID mouse. We found that there was no significant difference in the body weight between three groups (Fig. 2A), and no obvious abnormality of the mice after intraperitoneal injection of Compound 42 during the 39-day experiment. 14 days after transplantation, the tumors were clearly visible and could be measured. After 39 days, the tumor formation rate of High-dose group was lower than Low-dose group and control group, Low-dose group was lower than control group (Fig. 2B, C), and the tumor formation rate of High-dose group was less than $20 \%$ in CDX mouse model. Besides, the tumor size of High-dose group was smaller than other two groups $(P<$ 0.01), and tumor size of Low-dose group was smaller than control group $(P<0.05)$ (Fig. 2D). The same finding was observed in tumor weight of each group (Fig. 2E).

As for PDX mouse model, we obtained the clinical specimen for patient derived xenograft mouse model, the expression of KLK7 was confirmed in tumor tissue by IHC analysis (Fig. 3A). PDX mouse model was established by transplantation of pancreatic cancer patient tumor samples into nude mice (Fig. 3B). The same drug administration was taken as well as CDX model, and similar results were obtained. Indeed, the mouse weight change of three groups had no obvious difference during the 34-day treatment (Fig. 3C), the tumor formation rate (Fig. 3D, E), tumor size (Fig. 3F) and tumor weight (Fig. 3G) of High-dose group were much lower than other two groups. 
Besides, H\&E staining showed that no obvious damage and cancer metastasis was found in heart, liver, spleen, lung and kidney tissues in CDX and PDX mouse models (Sup Figure3).

These results suggest that treatment with Compound 42 significantly decreases pancreatic tumor growth rate, and high concentration of Compound 42 has better treatment effect.

\section{Compound 42 arrested pancreatic cancer cell cycle in $G_{0} / G_{1}$ phase without inducing cell apoptosis or necrosis}

It was demonstrated that Compound 42 inhibited pancreatic cell growth in vitro[5] and in vivo, to investigate the possible mechanism of action of Compound 42, the cell-cycle and apoptosis was analyzed by flow cytometry using PANC-1 and BxPC-3 cells treated with different concentration of Compound 42.

The result showed that the proportion of PANC-1 and BxPC-3 cells arrested in $\mathrm{G}_{0} / \mathrm{G}_{1}$ phase increased when increasing concentration of Compound 42, approximately $74.61 \%$ and $74.56 \%$ of PANC- 1 and BxPC-3 cells treated with $100 \mu \mathrm{M}$ of Compound 42 arrested in $\mathrm{G}_{0} / \mathrm{G}_{1}$ phase compared to $46.68 \%$ and $44.32 \%$ of these two cells treated by DMSO (Fig. 4A,B). In addition, it was demonstrated that the expression of Cyclin D1 and proliferating cell nuclear antigen (PCNA) was significantly reduced when treated with Compound 42 in PANC-1 and BxPC-3 cells (Fig. 4C, D). These results indicate that Compound 42 can arrest cell cycle in $G_{0} / G_{1}$ phase thus inhibiting cell proliferation. 
Then, apoptosis/necrosis assay was performed. Interestingly, the result showed that there was no obvious apoptosis or necrosis in PANC-1 and BxPC- 3 cells by any concentration of Compound 42 used in our experiment (Fig. 4E, F). In conclusion, we speculate that Compound 42 arrest PANC-1 and BxPC-3 cells into $\mathrm{G}_{0} / \mathrm{G} 1$ phase and inhibit cell proliferation without inducing cell apoptosis or necrosis.

\section{Compound 42 regulated the signal pathways related to cell proliferation and}

\section{ferroptosis}

To find out the inhibitory mechanism of Compound 42, transcriptome and proteome in PANC-1 cell treated with Compound 42 were analyzed.

Comparing with Control group, there were 755 genes expression changed when PANC-1 cell treated with $50 \mathrm{mM}$ of Compound 42, consisting of 437 up expression and 318 down expression (Fig.5A). When PANC-1 cell treated with $100 \mathrm{mM}$ of Compound 42, the quantity of different gene expression was much larger, containing 2303 up-regulation and 2272 down-regulation (Fig.5B). The similar finding was identified in different protein expression analysis, 211 up-regulation and 102 down-regulation when treated with $50 \mathrm{mM}$ of Compound 42, 1231 up-regulation and 2012 down-regulation when treated with $100 \mathrm{mM}$ of Compound 42 (Fig.5C, D).

To find tumor-specific inhibited pathways in PC by Compound 42, the KEGG signaling pathway enrichment analysis of transcriptome and proteome was established to estimate the main signaling pathways affected by Compound 42 treatment (Fig.5E, F). The result showed that a larger number of genes and proteins involved in cell proliferation and ferroptosis related pathways were enriched, especially the p53, cell 
cycle, Ferroptosis, protein digestion and absorption, ECM-receptor interaction and focal adhesion signaling pathways were closely related to cell growth of cancer.

It suggested that Compound 42 regulated the expression of genes and proteins in

PANC-1 cells to inhibit PANC-1 proliferation and induce ferroptosis by inhibiting KLK7 activity. In addition, the most important protein involved in ferroptosis is Glutathione peroxidase 4 (GPX4), and it was down-regulated to $72.8 \%$ in this experiment.

\section{Compound 42 down-regulated the expression of GPX4 protein to induce pancreatic cancer cells ferroptosis}

The above results suggested that the ferroptosis signaling pathway is involved in pancreatic cancer cell death treated by Compound 42, therefore, the core protein GPX4 in ferroptosis signaling pathway was then analyzed in this study.

The results showed that the expression of GPX4 in PANC-1 and BxPC-3 cells decreased when increasing the concentration of Compound 42 after $48 \mathrm{~h}$ treatment. (Fig.6A, B), But in HepG-2 cells, as lack of KLK7 expression, the expression of GPX4 was unchanged (Fig.6C). In addition, we found that expression of GPX4 in PANC-1 and BxPC-3 cells decreased gradually when treated with Compound 42 during a 72-hour assay, and BxPC-3 cells were more sensible to Compound 42 treatment compared to PANC-1 cells (Fig.6D, E). These results demonstrate that Compound 42 down-regulated expression of GPX4 in PANC-1 and BxPC-3 cells in both time and concentration dependent manner. 
Previous studies showed that Compound 42 has a good inhibitory effect on KLK7. In order to further clarify whether Compound 42 induces the down-regulation of GPX4 through KLK7 inhibition, we exogenously added wild-type PANC-1 cell culture medium to provide active KLK7 for rescue. The result showed that the expression of GPX4 of Compound 42 (Rescue) group were significantly higher than Compound 42 group, and higher than wild-type group (Fig.6F). This result indicated that Compound 42 reduced protein expression of GPX4 by targeting KLK7.

Moreover, we observed cell morphology change of PANC-1 and BxPC-3 cells after Compound 42 treatment using transmission electron microscope (TEM). The result showed that there were no obvious apoptotic body after Compound 42 treatment in PANC-1 cells for $72 \mathrm{~h}$ and BxPC-3 cells for $48 \mathrm{~h}$. In addition, we found that the mitochondria of PANC-1 cells were round and uneven in NC and DMSO groups. After treatment with Compound 42, the mitochondria in $50 \mu \mathrm{M}$ group began to be raptured and shortened, and the outer mitochondrial membranes was ruptured; the mitochondria in $100 \mu \mathrm{M}$ group became swelled, and the cristae disappeared and vacuoles were formed (Fig.6G). Further statistics analysis showed that the proportion of damaged mitochondria increased significantly when increasing Compound 42 concentration (Fig.6H). The same finding was observed in BxPC-3 cells (Fig.6I, J). The morphological changes of the mitochondria are consistent with cellular ferroptosis process.

\section{Compound 42 increased the concentration of iron ions and lipid ROS to induce}

\section{ferroptosis in pancreatic cancer cells}


The accumulation of lipid ROS is the key factor in ferroptosis[18]. Therefore, the evaluation of lipid ROS was performed in pancreatic cancer cells PANC-1 and BxPC-3. Accumulation of lipid ROS in BxPC-3 was significant after $24 \mathrm{~h}$ treatment by Compound 42, while accumulation of lipid ROS in PANC-1 cells became obvious after $48 \mathrm{~h}$ treatment (Fig.7A, B). Moreover, the accumulation of lipid ROS increased when increasing the concentration of Compound 42, which was consistent with the change trend of the GPX4 protein expression. However, there was no significant accumulation of lipid ROS in HepG-2 cells treated with Compound 42 after $24 \mathrm{~h}$ or $48 \mathrm{~h}$ (Fig.7C). In addition, we also analyzed total ROS in PANC-1 and BxPC-3 cells, the similar finding was observed as well as lipid ROS (Fig. 7D, E).

These results indicated that treatment with Compound 42 leads to accumulation of both lipid ROS and total ROS in PANC-1 and BxPC-3 cells through inhibiting KLK7 activity.

At present, there are two main pathways to induce ferroptosis. One pathway consists in decrease of expression of GPX4 protein or inhibit its activity can reduce the antioxidant capacity in the cell. The other pathway consists in oxidation of Poly-unsaturatedfattyacid (PUFA) which forms a large amount of lipid ROS to destroy the intracellular redox homeostasis and ultimately leads to ferroptosis[19].

Therefore, we further analyzed the iron ion concentration in pancreatic cancer cells, and we found that iron ion was accumulated in PANC-1 and BxPC-3 cells when increasing Compound 42 concentration after $48 \mathrm{~h}$ of treatment (Fig.7F, G). However, we also found that GSH and GSSG in PANC-1 and BxPC-3 cells were both increased, 
but the ratio between GSH/GSSG did not change significantly (Fig. 7H, I), in other words, the redox homeostasis was unbroken. This show that the effect of Compound 42 could activate the regulation of cell redox stress for drug resistance, but could not destroy the balance of redox. 


\section{Discussion}

High expression of KLK7 has been reported to be closely related to the malignant biological behavior of pancreatic cancer[3, 20, 21]. Zheng et al. proposed that KLK7 is an independent risk factor for vascular invasion of pancreatic cancer[4]. Through the analysis results based on TCGA database and GTEx database, we found that KLK7 was significantly high expressed in pancreatic cancer and ovarian cancer, and the high-expression of KLK7 was inversely proportional to long-term survival rate (>1.5 years) of pancreatic cancer patients. Therefore, targeting KLK7 to inhibit pancreatic cancer has great clinical significance.

At present, the existing KLK7 inhibitors include physiological and non-physiological inhibitors, the physiological inhibitors of KLK7 are mainly endogenous proteins, and the non-physiological inhibitors of KLK7 are peptide derivatives and small organic molecules[22]. These inhibitors have their own advantages and disadvantages in inhibitory effect and bioavailability. However, they are mainly reversible inhibitors of KLK7. Compared to irreversible inhibitors, the inhibitory effect of reversible inhibitors is limited[15]. Compound 42 is a substrate suicide inhibitor of KLK7, which was designed and synthesized in previous study[15]. Compound 42 contains a lactone structure which is unstable with ring-opening and activity loss in dilute alkaline condition (Sup Fig.4). Therefore, the stability of Compound 42 in PBS $(\mathrm{pH}=7.4)$ was evaluated before in vivo experiment. The result showed that Compound 42 was degraded very slowly at pH 7.4 (Sup Fig.5A, B). 
In our previous study, we found that $45 \mu \mathrm{M}$ of Compound 42 could inhibit the proliferation, migration and invasion ability of PANC-1 cell efficiently[5]. In this study, in order to avoid overestimated the anti-pancreatic cancer effect of Compound 42 in the following in vivo experiment, we mixed Compound 42 with cell culture medium for $6 \mathrm{~h}$ before treating PANC-1 and BxPC-3 cells. Compared to the previous study, the concentration of Compound 42 needed is higher in cell proliferation inhibition (Sup Fig.5C). In addition, we supposed the degradation of Compound 42 avoid the enrichment of this molecule in body, which can effectively reduce its side effects. Meanwhile, a 39-day toxicity assay was performed to determine the toxicity of Compound 42 before treatment assay in vivo, and we found no obvious toxicity of Compound 42 to mice in our study (Sup Fig.2).

In this study, we established the pancreatic cancer CDX and PDX mouse model and both successfully demonstrated inhibitory effect of Compound 42 in tumor formation and development of pancreatic cancer, use of high dose of Compound 42 had better therapeutic effect than low dose. CDX model is a traditional model used in cancer research, but the microenvironment of PDX model is more accuracy. It is worth mentioning that SCID mouse was used in CDX model, but nude mouse with thymus taking-off was used in PDX model, which was due to the growth rate and malignancy of cancer cell from patient specimen in our experiment was much higher than that of PANC-1 cells. These experiments confirmed the inhibitory effect of Compound 42 in pancreatic cancer cells, greatly enhancing its potency of the development of drug. 
Ferroptosis is a new non-apoptotic regulated cell death process[23], and it is different from other types of regulated cell death processes in cell morphology and biochemical characteristics[6, 23]. In this study, we found that in pancreatic cancer cell lines, the key protein in ferroptosis GPX4 was significantly down-regulated in a time and concentration-dependent manner with Compound 42 treatment. In addition, the content of intracellular iron ion, total ROS, lipid ROS and the proportion of damaged mitochondria were all obviously increased when giving Compound 42. However, due to the differences in drug tolerance and self-regulation capability in different pancreatic cancer cell lines, BxPC-3 cells were more sensible to treatment with Compound 42 compared to PANC-1 cells. In addition, in HepG-2 cells without KLK7 expression, cell growth was not inhibited by Compound 42, and expression of GPX4 protein and lipid ROS, the key indicators related to ferroptosis, did not changed. Taking together, these results demonstrate that Compound 42, a substrate suicide inhibitor KLK7, can inhibit pancreatic cell proliferation and induce the pancreatic cancer cells into ferroptosis.

However, we did not add iron exogenously into the culture medium in our experiment, which means that the accumulation of intracellular iron ions in PANC-1 cells and BxPC-3 cells treated by Compound 42 was caused by release of free iron ions from chelated iron ions. Further analysis of the proteomics results indicated that the intracellular ubiquitin-proteasome system was fully activated in PANC-1 cells after treated by Compound 42, the similar result was found in RNAseq analysis. Therefore, we speculate that Compound 42 activates the PANC-1 cells 
ubiquitin-proteasome degradation system through an unknown pathway, thereby reducing the expression of GPX4 and increasing the content of free iron ions to induce ferroptosis in pancreatic cancer cells.

To further clarify the important function of KLK7 in the development of pancreatic cancer, we will study the exact role of KLK7 in induction of ferroptosis in pancreatic cancer in the following research, and it will provide us a reliable theoretical basis to promote the development of drugs targeting KLK7 for the treatment of pancreatic cancer. 


\section{Materials and Methods}

\section{Analysis of $K L K 7$ expression in patients with pancreatic cancer}

To analyze the transcription of $K L K 7$ gene in various cancers, Cancer Genome Atlas (TCGA) database and Genotype-Tissue Expression (GTEx) were utilized, expression of mRNA of KLK7 was analyzed using Sangerbox bioinformatics analysis tool between normal and cancer tissues. In addition, the TCGA Pancreatic Cancer (PAAD) was analyzed using UCSC Xena analysis tool for the relationship between KLK7 expression and survival rate of pancreatic cancer patients.

\section{Cell cultures}

Human pancreatic cancer cell lines PANC-1, BxPC-3 and Human hepatocarcinoma cell line HepG-2 were obtained from Shanghai Zhong Qiao Xin Zhou Biotechnology Co. Ltd. (China). These cells were cultured in DMEM (Gibco, USA) containing $10 \%$ fetal bovine serum (Gibco, USA) and $1 \%$ penicillin-streptomycin mixture (Biosharp, China), incubated in $5 \% \mathrm{CO}_{2}$ at $37^{\circ} \mathrm{C}$.

\section{Cell viability CCK-8 assay}

PANC-1, BxPC-3 and HepG-2 cells were seeded into 96-well microplate with $4 \times 10^{3}$ cells per well in DMEM medium for $12 \mathrm{~h}$, then incubated with different final concentration $(0 \mu \mathrm{M}, 25 \mu \mathrm{M}, 50 \mu \mathrm{M}, 75 \mu \mathrm{M}, 100 \mu \mathrm{M})$ of Compound 42 for $48 \mathrm{~h}$. Cells were washed and incubated according to the guidance of CCK-8 KIT (Biosharp, China). The absorbance of each well at $450 \mathrm{~nm}$ was measured. Each experiment was repeated three times.

\section{Clinical specimen}


Human pancreatic cancer tissue used to establish the patient derived xenograft (PDX) mouse model and liver cancer tissues were obtained from the First College of Clinical Medical Science of China Three Gorges University without chemotherapy and radiotherapy. The access of clinical tissue obeyed the Clinical Ethics Committee of China Three Gorges University.

\section{Animals}

SCID mice (4-week-old, 14- 16g) and nude mice (4-week-old, 16 - $18 \mathrm{~g}$ ) were obtained from Beijing Huafukang bioscience Co.INC (China) for in vivo experiment. The experimental procedures were carried out according to the Ethics Committee for Experiments on Animals of Laboratory Animal Center of China Three Gorges University.

\section{CDX mouse model}

$2 \times 10^{6}$ of PANC- 1 cells were collected and injected into both sides of scapula of SCID mice. After inoculation for 3 days, the mice were randomly divided into control group, low-dose group $(17.0 \mu \mathrm{g} / \mathrm{g})$ and high-dose group $(68.2 \mu \mathrm{g} / \mathrm{g})$, consisting of 7 tumor-bearing mice per group. DMSO and Compound $42(80 \mu \mathrm{L})$ were intraperitoneal injected into control group and Compound 42 groups every 2 days for 39 days.

\section{PDX mouse model}

Clinical specimen of pancreatic cancer was obtained and cut into $2 \times 2 \times 2 \mathrm{~mm}$ piece, then transplanted into both sides of scapula of nude mice. After transplantation for 3 days, the mice were randomly divided into control group, low-dose group (17.0 
$\mu \mathrm{g} / \mathrm{g})$ and high-dose group $(68.2 \mu \mathrm{g} / \mathrm{g})$, consisting of 6 tumor-bearing mice per group. DMSO and Compound $42(80 \mu \mathrm{L})$ were intraperitoneal injected into control group and Compound 42 groups every 2 days for 35 days.

\section{Analysis of the in vivo anti-tumor effect of Compound 42}

The behaviors of each mouse such as tumor formation rate, tumor volume and mice weight were noted every 2 days. Tumor volume was calculated according to the formula: Volume $=1 / 2 \times$ length $\times$ width $^{2}$. The mice were sacrificed after experiment and the tumor weight were measured. The tissues of tumor, heart, liver, spleen, lung, kidney were obtained for $\mathrm{H} \& \mathrm{E}$ staining.

\section{Cell cycle assay}

The distribution of cells in sub- $\mathrm{G}_{1}, \mathrm{G}_{0} / \mathrm{G}_{1}, \mathrm{~S}$ and $\mathrm{G}_{2} / \mathrm{M}$ multi-nuclear phases were analyzed using BD FACSVerse Flow Cytometer (BD Biosciences, USA). PANC-1 and BxPC-3 cells were seeded into 6-well microplate with $1 \times 10^{6}$ cells per well in DMEM medium for $12 \mathrm{~h}$, then added Compound 42 pre-incubated with cell culture medium with different final concentration $(0 \mu \mathrm{M}, 50 \mu \mathrm{M}, 75 \mu \mathrm{M}, 100 \mu \mathrm{M})$ for $48 \mathrm{~h}$. Cells were washed and then resuspended in $500 \mu \mathrm{L}$ of PI/RNase Staining Solution (BD Biosciences, USA). After incubated on ice for $30 \mathrm{~min}$, cells were analyzed using flow cytometer and FACSuite software.

\section{Western Blot assay}

The detailed steps were described in previous publication[24]. The primary monoclonal antibodies included anti-PCNA (Beyotime Biotechnology, China), anti-Cyclin D1 (Beyotime Biotechnology, China), anti-GPX4 (Abcam, UK) and 
anti-GAPDH (Biosharp, China). The secondary antibodies are Goat anti-mouse IgG (TransGen Biotech, China) and goat anti-rabbit IgG (TransGen Biotech, China)

\section{Apoptosis/Necrosis assay}

Cell apoptosis and necrosis was analyzed using BD FACSVerse Flow Cytometer. PANC-1 and BxPC-3cells were seeded into 6-well microplate with $1 \times 10^{6}$ cells per well in DMEM medium for $12 \mathrm{~h}$, then incubated with different final concentration (0 $\mu \mathrm{M}, 50 \mu \mathrm{M}, 100 \mu \mathrm{M})$ of Compound 42 for $24 \mathrm{~h}(\mathrm{BxPC}-3)$ or $48 \mathrm{~h}$ (PANC-1). Cells were collected and washed, and stained with Cell Apoptosis and Necrosis Analysis Kit (KeyGEN BioTECH, China), then analyzed by flow cytometry. Each experiment was repeated three times.

\section{Lipid peroxidation assay}

PANC-1, BxPC-3and HepG2 cells were seeded into 6-well microplate with $1 \times 10^{6}$ cells per well in DMEM medium for $12 \mathrm{~h}$, then incubated with different final concentration $(0 \mu \mathrm{M}, 50 \mu \mathrm{M}, 100 \mu \mathrm{M})$ of Compound 42 for $24 \mathrm{~h}$ and $48 \mathrm{~h}$, respectively. Cells were collected and washed once with PBS, and resuspended in 900 $\mu \mathrm{L}$ DMEM medium with $2 \mu \mathrm{M}$ C11 BODIPY 581/59 (Shanghai Mao Kang biotechnology, China). After incubated in dark at $37^{\circ} \mathrm{C}$ for 30 mins, cells were washed twice with PBS, then stained with $0.1 \mu \mathrm{g} / \mathrm{mL}$ PI at $37^{\circ} \mathrm{C}$ for $5 \mathrm{~min}$. Finally, Cells were collected and washed once with PBS, then analyzed by flow cytometry. Each experiment was repeated three times.

\section{Total ROS assay}


PANC-1 and BxPC-3 cells were seeded into 6-well microplate with $1 \times 10^{6}$ cells per well in DMEM medium for $12 \mathrm{~h}$, then incubated with different final concentration $(0 \mu \mathrm{M}, 50 \mu \mathrm{M}, 100 \mu \mathrm{M})$ of Compound 42 for $24 \mathrm{~h}$ and $48 \mathrm{~h}$, respectively. Cells were collected and washed once with PBS, and resuspended in $400 \mu \mathrm{L}$ DMEM medium with $10 \mu \mathrm{M} / \mathrm{L}$ DCFH-DA (Beyotime Biotechnology, China). After incubated in dark at $37{ }^{\circ} \mathrm{C}$ for 30 mins, cells were washed twice with PBS, and then stained with 0.1 $\mu \mathrm{g} / \mathrm{mL}$ PI at $37^{\circ} \mathrm{C}$ for 5 min. Finally, Cells were collected and washed once with PBS, then analyzed by flow cytometry. Each experiment was repeated three times.

\section{Iron Ion assay}

PANC-1 and BxPC-3 cells were seeded into $100 \mathrm{~mm}$ cell culture dish with $1 \times 10^{7}$ cells per well in DMEM medium for $12 \mathrm{~h}$, then incubated with different final concentration $(0 \mu \mathrm{M}, 50 \mu \mathrm{M}, 100 \mu \mathrm{M})$ of Compound 42 for $24 \mathrm{~h}(\mathrm{BxPC}-3)$ or $48 \mathrm{~h}$ (PANC-1). Cells were collected and counted, then washed twice with ice-cold PBS. Cells were treated according to the guidance of Iron Detection Kit instruction (APPLYGEN, China), the absorbance was measured at $550 \mathrm{~nm}$. The concentration of iron ion was evaluated using standard curve. Each experiment was repeated three times.

\section{GSH/GSSG assay}

PANC-1 and BxPC-3 cells were seeded into $100 \mathrm{~mm}$ cell culture dish with $1 \times 10^{7}$ cells per well in DMEM medium for $12 \mathrm{~h}$, then incubated with different final concentration $(0 \mu \mathrm{M}, 50 \mu \mathrm{M}, 100 \mu \mathrm{M})$ of Compound 42 for $24 \mathrm{~h}(\mathrm{BxPC}-3)$ or $48 \mathrm{~h}$ (PANC-1). Cells were collected and counted, then washed twice with ice-cold PBS. 
Cells were treated according to the guidance of GSH kit (Solarbio, China) and GSSG

kit (Solarbio, China), the absorbance was measured at $412 \mathrm{~nm}$. The concentration of

GSH and GSSG was evaluated using the standard curve. Each experiment was repeated three times.

\section{TEM}

PANC-1 and BxPC-3 cells were seeded into 6-well microplate with $1 \times 10^{6}$ cells per well in DMEM medium for $12 \mathrm{~h}$, then incubated with different final concentration $(0 \mu \mathrm{M}, 50 \mu \mathrm{M}, 100 \mu \mathrm{M})$ of Compound 42 for $48 \mathrm{~h}(\mathrm{BxPC}-3)$ and $72 \mathrm{~h}$ (PANC-1), respectively. Cells were collected and counted, then washed and fixed with $2.5 \%$ glutaraldehyde solution at $4^{\circ} \mathrm{C}$. Then, cells were dehydrated, embedded, and sliced. The intracellular mitochondria were observed under transmission electron microscope.

\section{Rescue assay}

PANC-1 cells (divided into four groups: Wild, Wild (resucue), C42 and C42 (rescue)) were seeded into $60 \mathrm{~mm}$ cell culture dish with $2 \times 10^{6}$ cells per well in DMEM medium for 12 h. C42 and C42(rescue) groups were incubated with $100 \mu \mathrm{M}$ of Compound 42 for $48 \mathrm{~h}$. The wild group was collected and ultrafiltrated to obtain the supernatant, then resuspended into $6 \mathrm{~mL}$ with complete DMEM medium as the rescue culture medium. Wild and $\mathrm{C} 42$ groups were added with $3 \mathrm{~mL}$ of DMEM medium, wild(rescue) and $\mathrm{C} 42$ (rescue) groups were added with $3 \mathrm{~mL}$ of rescue culture medium. After culturing for $20 \mathrm{~h}$, cells were collected and counted. The total 
cell protein of four groups were prepared for protein expression analysis using Western Blot.

\section{RNA isolation and RNA-seq analysis}

PANC-1 cells were divided into 3 groups (DMSO, $50 \mu \mathrm{M}$ and $100 \mu \mathrm{M}$ of Compound 42) with three replicate per group. After $48 \mathrm{~h}$ incubation, total RNA was extracted using TRIzol@Reagent (Invitrogen, USA). RNA concentration and insert size of library were assessed, and the cluster of the index-coded samples was performed on a cBot cluster generation system. After cluster generation, the libraries were sequenced using Illumina platform and $150 \mathrm{bp}$ paired-end reads were generated. The data after quality control was used for quantitative analysis of transcripts, the different expression of transcripts between samples was analyzed. Finally, functional annotation and KEGG pathway analysis of differently expressed genes were performed.

\section{TMT quantitative proteomics analysis}

PANC-1 cells were divided into 3 groups (DMSO, $50 \mu \mathrm{M}$ and $100 \mu \mathrm{M}$ of Compound 42) with three replicate per group. After $48 \mathrm{~h}$ incubation, cells were collected for protein extraction, trypsin digestion, labeling peptides were performed according to the manufacturer's protocol for TMT kit (Thermo Fisher Scientific, Waltham, MA, USA). The tryptic peptides were fractionated by high $\mathrm{pH}$ reverse-phase HPLC (Thermo Fisher Scientific, Waltham, MA, USA) and analyzed by LC-MS/MS (Thermo Fisher Scientific, Waltham, MA, USA), the generated 
MS/MS data were processed using Maxquant search engine (v.1.5.2.8), FDR was adjusted to $<1 \%$ and minimum score for modified peptides was set to $>40$.

\section{Statistics}

The Student's t test was used to determine the statistical significance of data. The $P$ value was considered to be significant. Data presented in the figures represented the mean \pm standard error.

\section{Conflict of interest}

No conflict of interest exits in the submission of this manuscript, and manuscript is approved by all authors for publication. I would like to declare that the work described was original research that has not been published previously, and not under consideration for publication elsewhere.

\section{Acknowledgements}

This study was supported by The First College of Clinical Medical Science, China Three Gorges University. This work was financially supported by the National Natural Science Foundation of China (81602743, 81402568), the Scientific Research Fund of the Hunan Provincial Education Department (D20191203), open fund of Hubei Key Laboratory of Tumor Microenvironment and Immunotherapy (Three Gorges University) (2019KZL06、2019KZL02、2020KZL02), and open fund of Hunan Key Laboratory of Hengyang Normal College (NY20K02). 


\section{References}

1. Martens S, Lefesvre P, Nicolle R, Biankin AV, Puleo F, Van Laethem JL, Rooman I: Different shades of pancreatic ductal adenocarcinoma, different paths towards precision therapeutic applications. Annals of Oncology 2019, 30 (9) :1428-1436.

2. Zhao C, Gao F, Li Q: The Distributional Characteristic and Growing Trend of Pancreatic Cancer in China. Pancreas 2019, 48 (6) :E56-E56.

3. Iakovlev V, Siegel ER, Tsao M-S, Haun RS: Expression of Kallikrein-Related Peptidase 7 Predicts Poor Prognosis in Patients with Unresectable Pancreatic Ductal Adenocarcinoma. Cancer Epidemiology Biomarkers \& Prevention 2012, 21 (7) :1135-1142.

4. Zheng SL, Feng MY, Yang G, Xiong GB, Zhao YP: The expression of KLK7 in pancreatic cancer and the effects on the biological behavior of pancreatic cancer cells. Zhonghua wai ke za zhi 2018, 56 (5) : 391-397.

5. Du JP, Li L, Zheng J, Zhang D, Liu W, Zheng WH, Li XS, Yao RC, Wang F, Liu S et al: Kallikrein-related peptidase 7 is a potential target for the treatment of pancreatic cancer. In: Oncotarget. vol. 9; 2018: 12894-12906.

6. Dixon, Scott J: Ferroptosis: bug or feature? Immunological Reviews 2017, 277 (1) : 150-157.

7. Stockwell BR, Jiang X: A Physiological Function for Ferroptosis in Tumor Suppression by the Immune System. Cell Metabolism 2019, 30 (1) : 14-15.

8. Chen G, Guo G, Zhou X, Chen H: Potential mechanism of ferroptosis in pancreatic cancer (Review). Oncology Letters 2020, 19 (1) : 579-587.

9. Lu B, Chen XB, Ying MD, He QJ, Cao J, Yang B: The Role of Ferroptosis in Cancer Development and Treatment Response. Front Pharmacol 2017, 8:992.

10. Katsori A-M, Hadjipavlou-Litina D: Coumarin derivatives: an updated patent review (2012-2014). Expert Opinion on Therapeutic Patents 2014, 24(12) : 1323-1347.

11. Perumalsamy H, Sankarapandian K, Veerappan K, Natarajan S, Kandaswamy N, Thangavelu L, Balusamy SR: In silico and in vitro analysis of coumarin derivative induced anticancer effects by undergoing intrinsic pathway mediated apoptosis in human stomach cancer. Phytomedicine 2018, 46:119-130.

12. Zhao JW, Wu ZH, Guo JW, Huang MJ, You YZ, Liu HM, Huang LH: Synthesis and anti-gastric cancer activity evaluation of novel triazole 
nucleobase analogues containing steroidal/coumarin/quinoline moieties. Eur J Med Chem 2019, 181:111520.

13. Zhang L, Xu Z: Coumarin-containing hybrids and their anticancer activities. Eur J Med Chem 2019, 181:111587.

14. Zhang Y, Song N, Li Y, Yang Z, Chen L, Sun T, Xie Z: Comparative study of two near-infrared coumarin-BODIPY dyes for bioimaging and photothermal therapy of cancer. $J$ Mater Chem $B$ 2019, 7 (30) : 4717-4724.

15. Tan X, Soualmia F, Furio L, Renard J-F, Kempen I, Qin L, Pagano M, Pirotte B, El Amri C, Hovnanian A et al: Toward the First Class of Suicide Inhibitors of Kallikreins Involved in Skin Diseases. Journal of Medicinal Chemistry 2015, 58(2):598-612.

16. Zheng X, He M, Tan X, Zheng J, Wang F, Liu S: 3D-quantitative structure-activity relationship and docking studies of coumarin derivatives as tissue kallikrein 7 inhibitors. J Pharm Pharmacol 2017, 69 (9) : 1136-1144.

17. Johnson SK, Ramani VC, Hennings L, Haun RS: Kallikrein 7 enhances pancreatic cancer cell invasion by shedding E-cadherin. Cancer 2007, 109 (9) : 1811-1820.

18. Hirschhorn T, Stockwell BR: The development of the concept of ferroptosis. Free Radic Biol Med 2019, 133:130-143.

19. Dixon SJ, Patel DN, Welsch M, Skouta R, Lee ED, Hayano M, Thomas AG, Gleason CE, Tatonetti NP, Slusher BS et al: Pharmacological inhibition of cystine-glutamate exchange induces endoplasmic reticulum stress and ferroptosis. Elife 2014, 3:e02523.

20. Dorn J, Gkazepis A, Kotzsch M, Kremer M, Propping C, Mayer K, Mengele K, Diamandis EP, Kiechle M, Magdolen V et al: Clinical value of protein expression of kallikrein-related peptidase 7 (KLK7) in ovarian cancer. Biological Chemistry 2014, 395 (1):95-107.

21. Walker F, Nicole P, Jallane A, Soosaipillai A, Mosbach V, Oikonomopoulou K, Diamandis EP, Magdolen V, Darmoul D: Kallikrein-related peptidase 7 (KLK7) is a proliferative factor that is aberrantly expressed in human colon cancer. Biological Chemistry 2014, 395 (9) : 1075-1086.

22. Goettig P, Magdolen V, Brandstetter $\mathrm{H}$ : Natural and synthetic inhibitors of kallikrein-related peptidases (KLKs). Biochimie 2010, 92 (11) : 1546-1567.

23. Dixon SJ, Lemberg KM, Lamprecht MR, Skouta R, Zaitsev EM, Gleason CE, Patel DN, Bauer AJ, Cantley AM, Yang WS et al: Ferroptosis: an iron-dependent form of nonapoptotic cell death. Cell 2012, 149 (5) : 1060-1072.

24. Zhao J, Wang Y, Liang Q, Xu Y, Sang J: MAGEA1 inhibits the expression of BORIS via increased promoter methylation. Journal of Cell Science 2019, 132 (1). 
bioRxiv preprint doi: https://doi.org/10.1101/2021.09.27.461898; this version posted September 27, 2021. The copyright holder for this preprint (which was not certified by peer review) is the author/funder, who has granted bioRxiv a license to display the preprint in perpetuity. It is made available under aCC-BY-NC-ND 4.0 International license. 
A

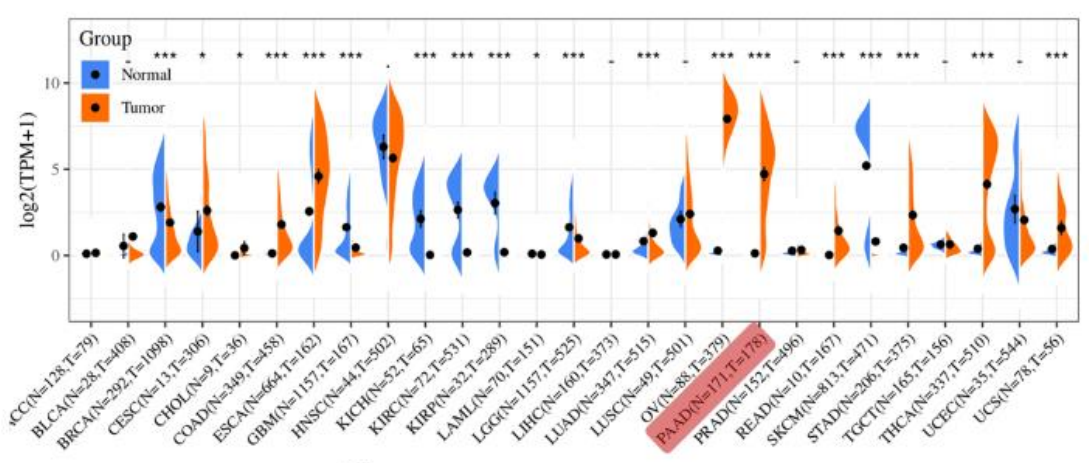

B

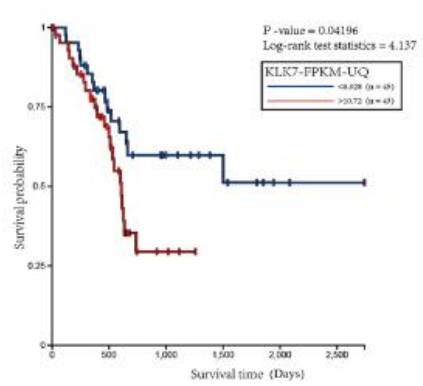

D

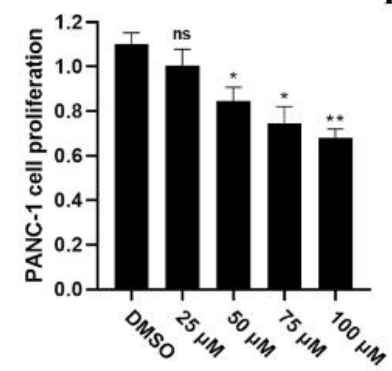

C

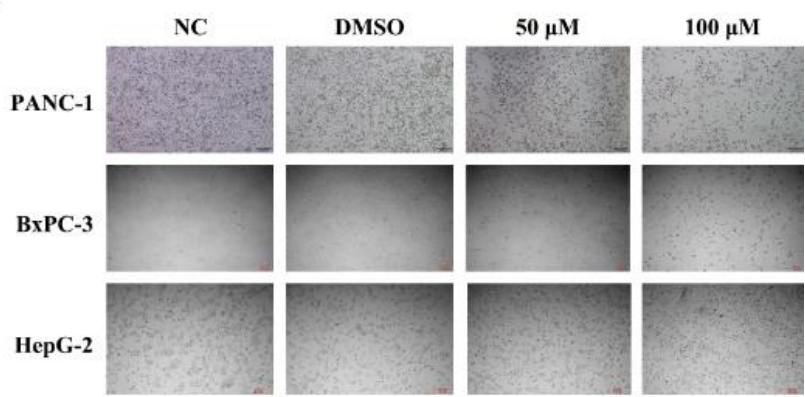

$\mathbf{E}$

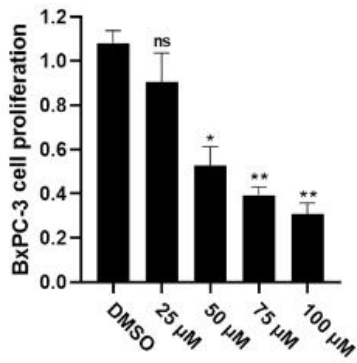

F

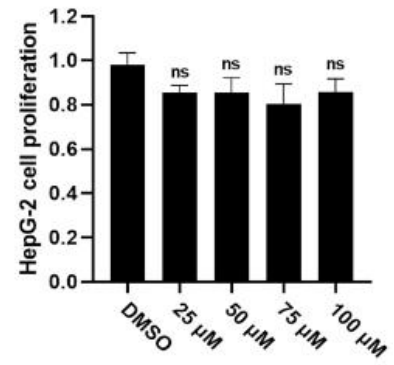

Figure 1. KLK7 plays an important role in pancreatic cancer (PC) and Compound 42 suppresses cell vitality of PANC-1 and BxPC-3 cells in a dose-dependent manner. A: The transcription of KLK7 gene in pancreatic cancer tissue was selectively high expressed compared to normal tissue. B: The long-term ( $>1.5$ years) survival rate of pancreatic cancer patients was negatively correlated with KLK7 expression. C: Representative morphological changes of cells were observed under microscopy. D-F: Analysis of PANC-1 (D), BxPC-3 (E) and HepG-2 (F) cell viability when treated with different concentration of Compound 42 for 48 h. ns: no significant difference; *: $P<0.05 ; * *: P<0.01$. 
A

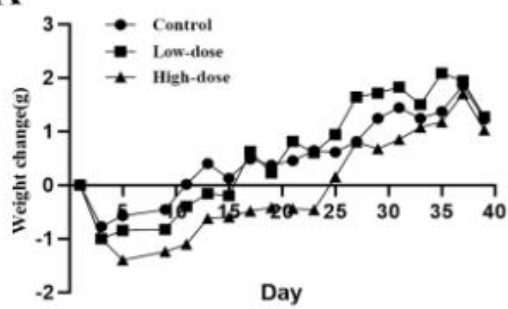

C

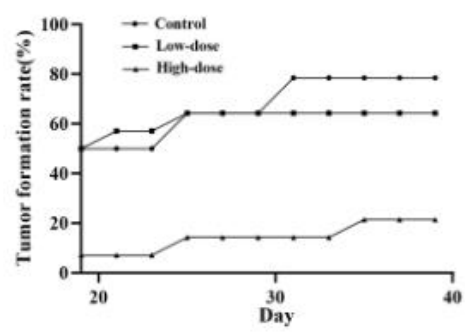

B

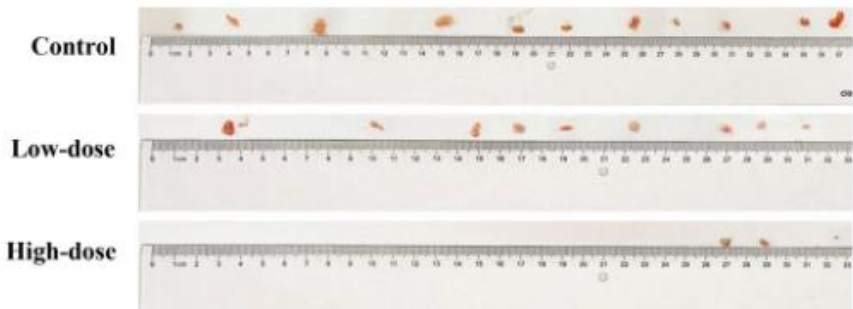

E

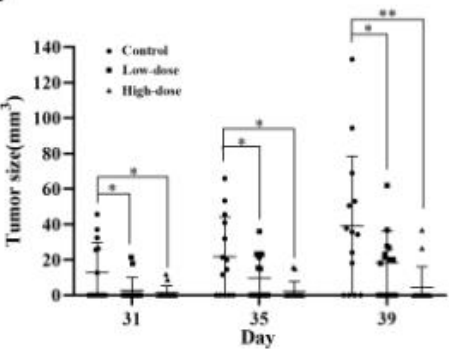

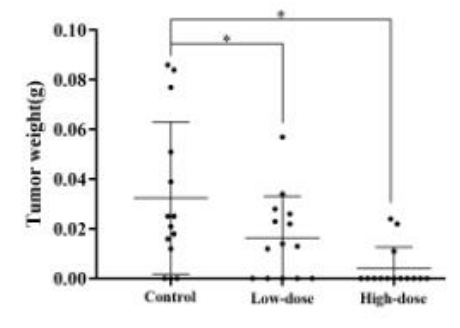

Figure 2. Compound 42 significantly inhibited the growth of pancreatic cancer in CDX

mouse model. A: The SCID mice injected with high dose $(68.2 \mu \mathrm{g} / \mathrm{g})$ and low dose $(17 \mu \mathrm{g} / \mathrm{g})$ of Compound 42 had no obvious weight difference compared to control group (n=7). B, C: Compound 42 inhibited tumor formation rate. D, E: The volume and weight of tumor was much smaller after treated with high-dose of Compound 42 compared to low dose group and control group. *: $P<0.05 ; * *: P<0.01$. 
A

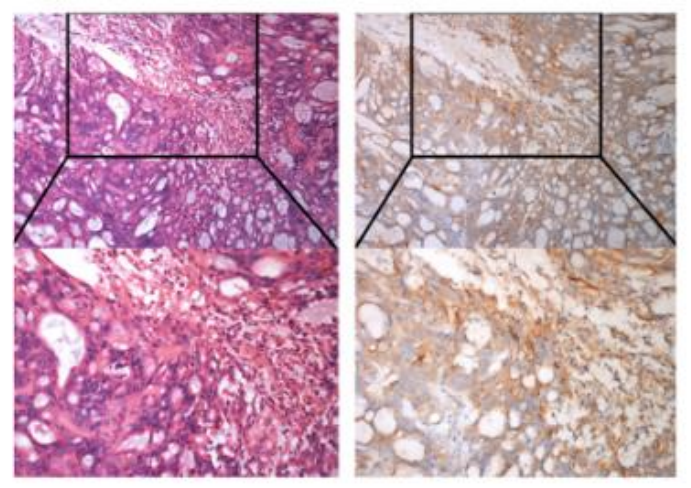

D

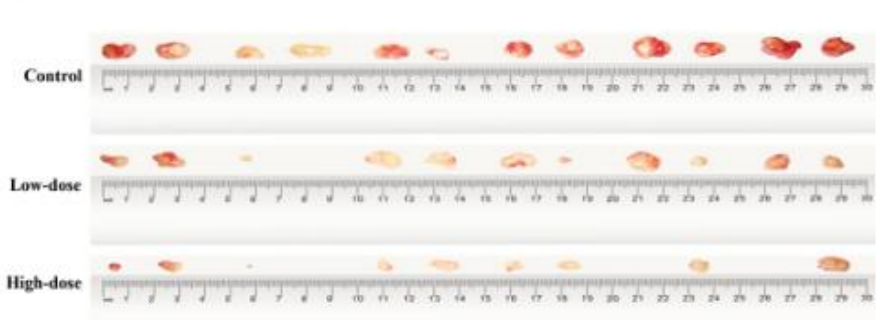

$\mathbf{F}$

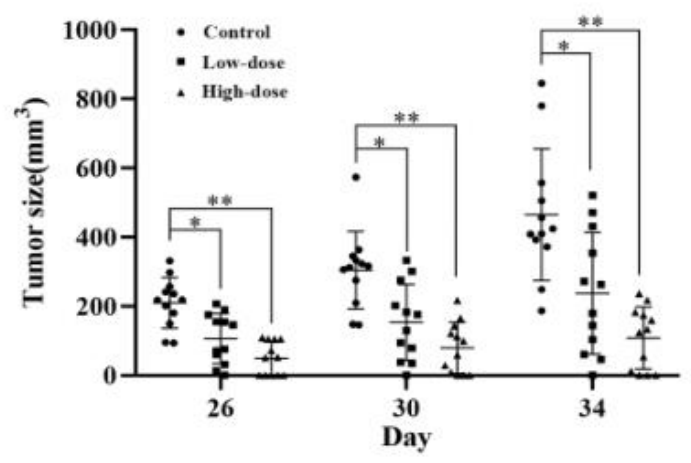

B

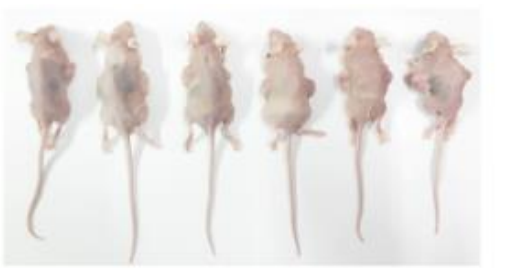

C

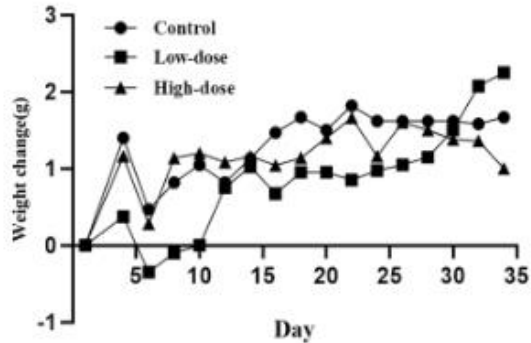

E

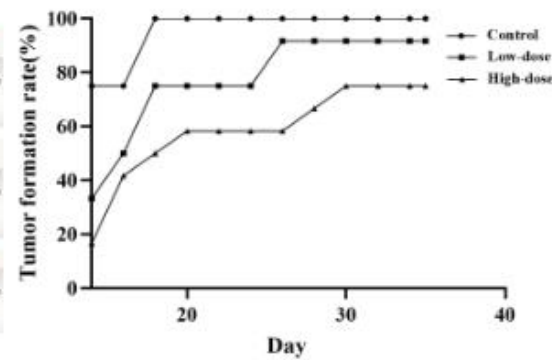

G

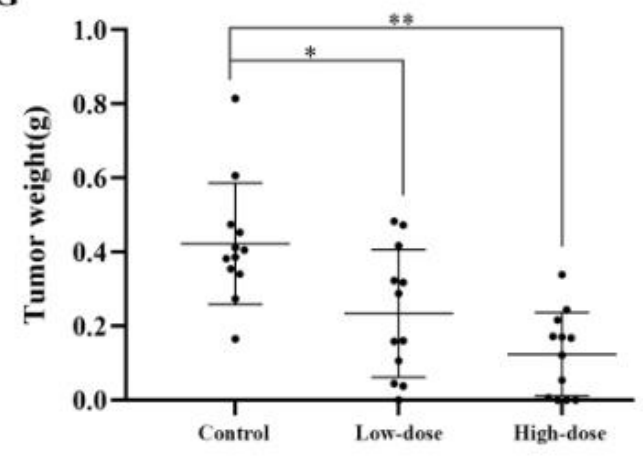

Figure 3. Compound 42 significantly inhibited the growth of pancreatic cancer in PDX

mouse model. A: The clinical specimen section for PDX mouse model was identified as high expression of KLK7 using anti-KLK7 immunohistochemistry assay. (D: 100x, F: 200×). B: Subcutaneous transplantationof clinical specimen of pancreatic cancer into nude mice for establishing PDX mouse model of pancreatic cancer. $(n=6) C$ : The body weight change of mice in 3 groups had no obvious difference. D, E: The tumor formation of PDX model was inhibited by Compound 42. F, G: Compound 42 inhibited the tumor growth in PDX model. *: $P<0.05 ; * *: P<0.01$. 
A

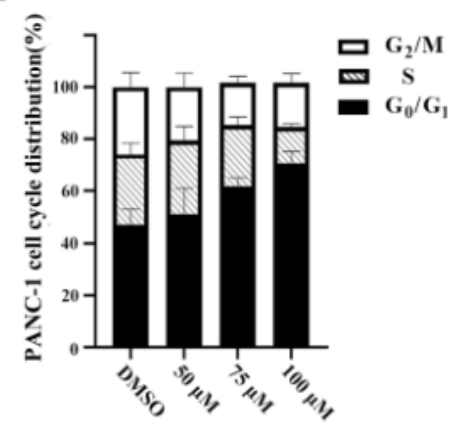

B

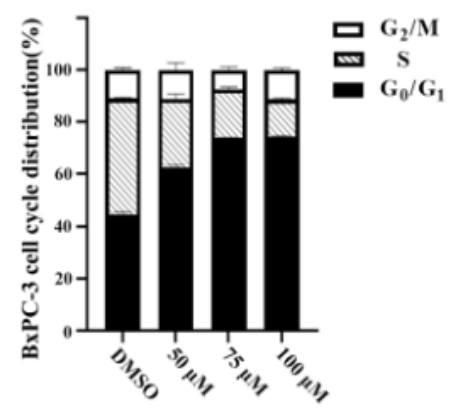

$\mathbf{E}$

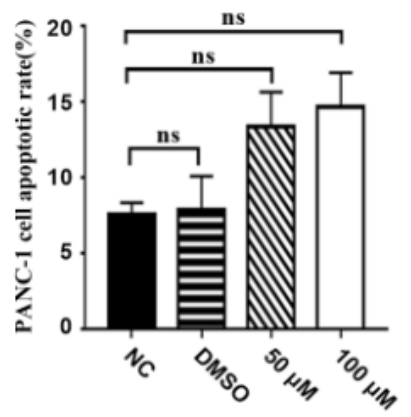

D
C
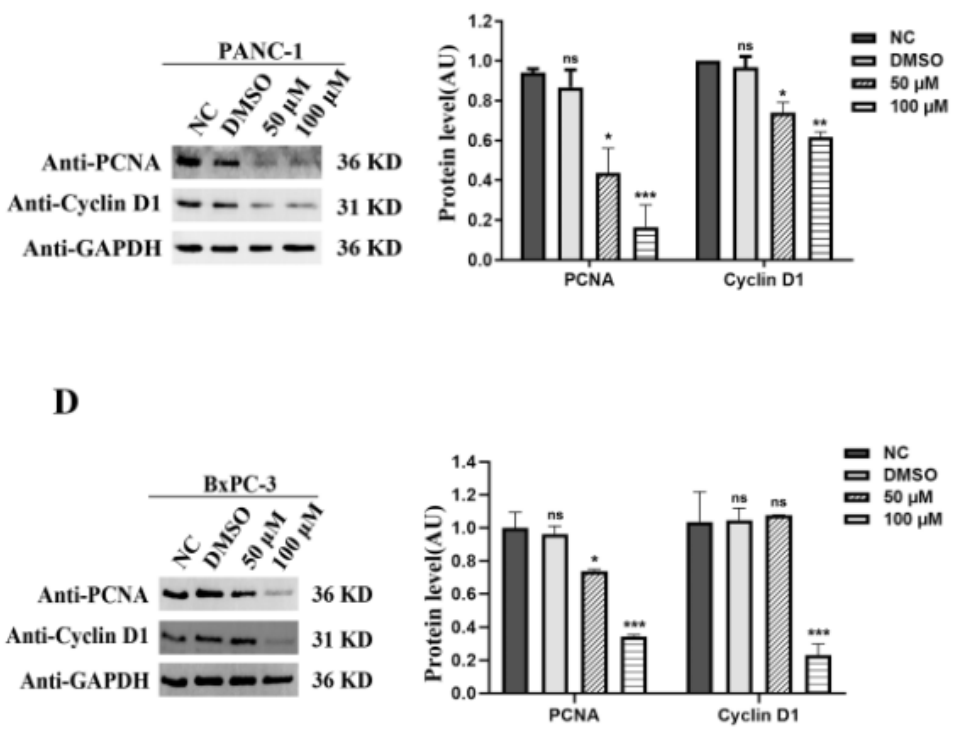

F

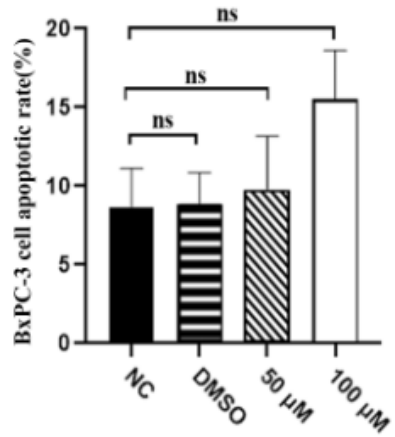

Figure 4. Compound 42 arrested PANC-1 cell cycle at G0/G1 phase without induction of cell apoptosis or necrosis. A - D: PANC-1 $(\mathrm{A}, \mathrm{C})$ and BxPC-3 (B, D) cells were treated with different concentration of Compound 42 for $48 \mathrm{~h}$ (DMSO-only, $50 \mu \mathrm{M}, 75 \mu \mathrm{M}$, and $100 \mu \mathrm{M}$ ), respectively. Compound 42 arrested cell cycle at $\mathrm{G}_{0} / \mathrm{G} 1$ phase in a concentration dependent manner. E, F: Western blot analysis of anti-PCNA and anti-Cyclin D1 of PANC-1 (E) and BxPC-3 (F) cells showed that the expression of PCNA and Cyclin D1 proteins was negatively correlated with concentration of Compound 42 used. H-G: PANC-1 (H) and BxPC-3 (G) cells were treated with Compound 42 for 48 h $(\mathrm{H}), 24 \mathrm{~h}(\mathrm{G})$, and Compound 42 did not induce 
bioRxiv preprint doi: https://doi.org/10.1101/2021.09.27.461898; this version posted September 27, 2021. The copyright holder for this preprint (which was not certified by peer review) is the author/funder, who has granted bioRxiv a license to display the preprint in perpetuity. It is made available under aCC-BY-NC-ND 4.0 International license.

obvious apoptosis or necrosis in these two pancreatic cancer cell lines. ns: no significant difference; *: $P<0.05 ; * *: P<0.01 ; * * *: P<0.001$. 
A

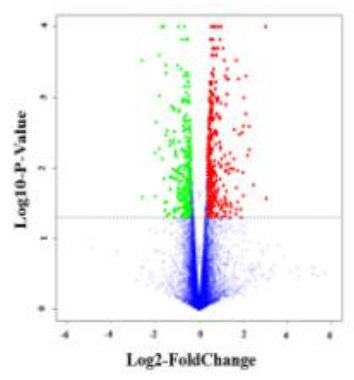

$\mathbf{E}$
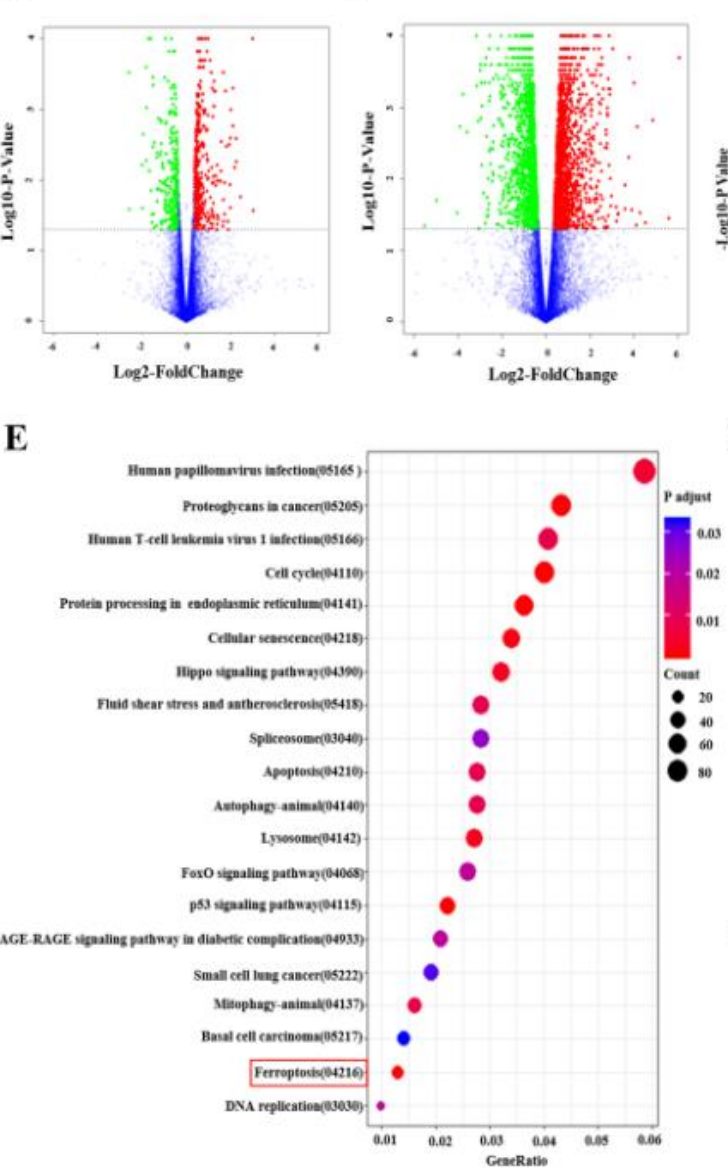

C

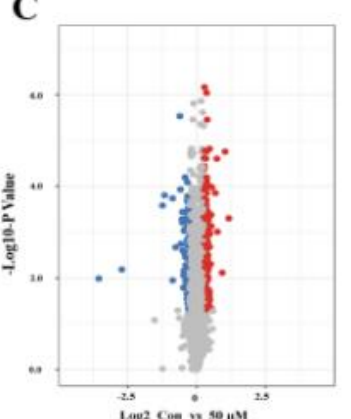

D

F

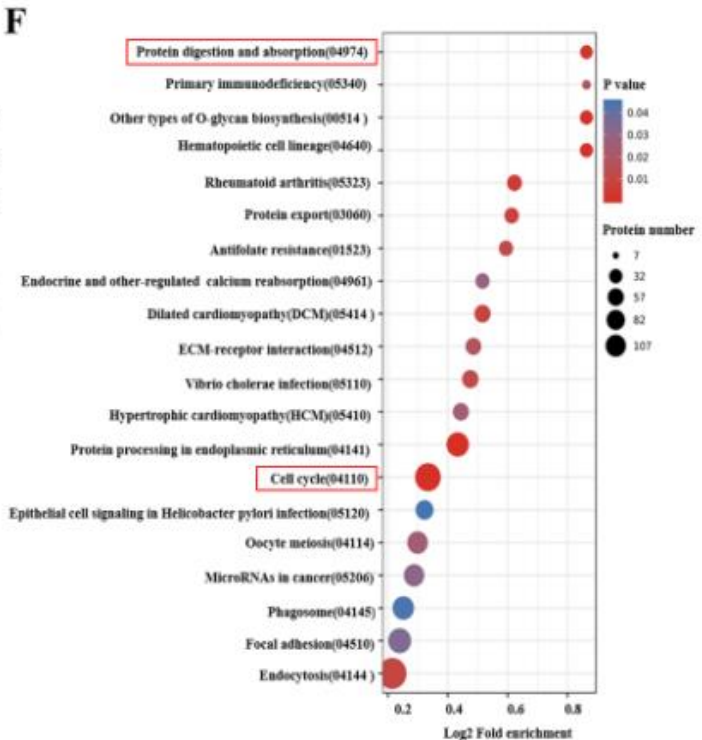

Figure 5. Genes and proteins regulated by Compound 42 and KEGG pathway enrichment analysis of differently expressed genes and proteins identified in Compound

42 treatment. A. B: Volcano plots of differently expressed genes in Control vs $50 \mu \mathrm{M}$ of Compound 42 (A) and Control vs $100 \mu \mathrm{M}$ of Compound 42 (B). C. D: Volcano plots of differently expressed proteins in Control vs $50 \mu \mathrm{M}$ of Compound 42 (C) and Control vs 100 $\mu \mathrm{M}$ of Compound 42 (D). E. F: The top 20 of KEGG pathways enriched in gene expression (E) and protein expression (F) in Control vs $100 \mu \mathrm{M}$ of Compound 42. The size of circles represented the quantity of differently expressed genes and proteins, the color of circles represented the $P$-adjust. The different $p$-adjustcutoff $(0.05)$ was used to assess the dependence of the significant pathway identification on $p$-adjust. 
A
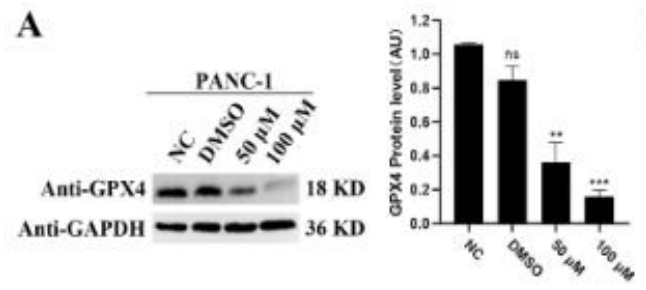

C

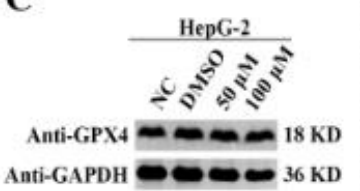

E

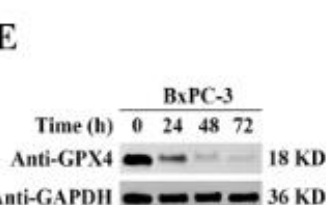

G

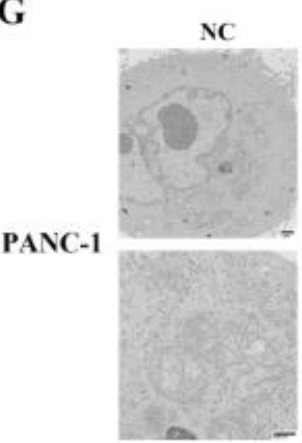

I
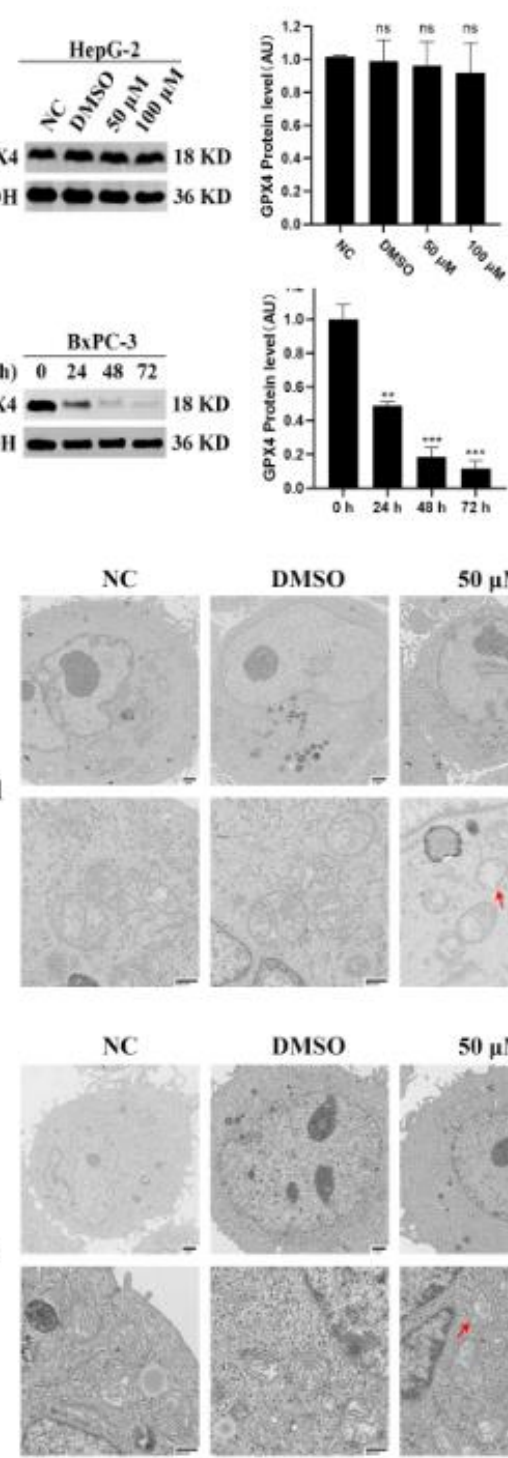

DMSo
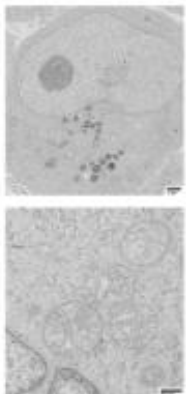

DMSO

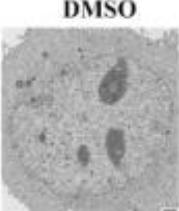

BxPC-3

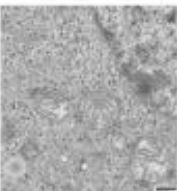

B
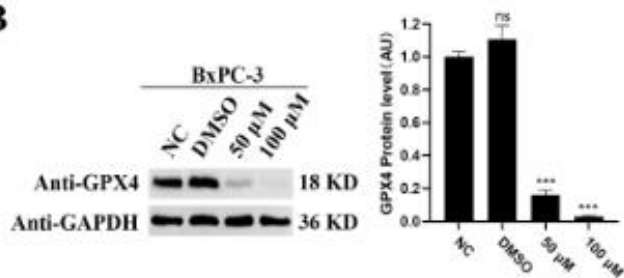

D
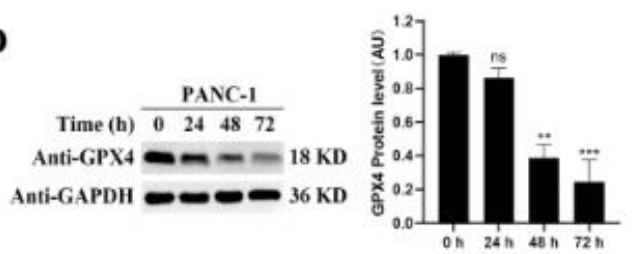

F
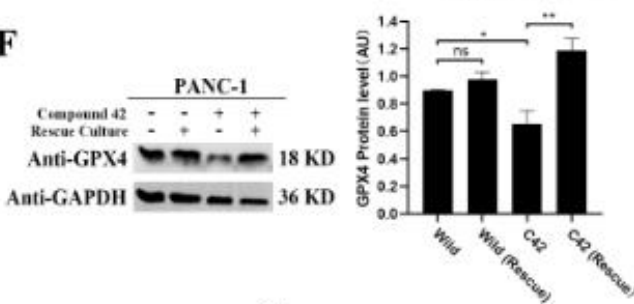

H

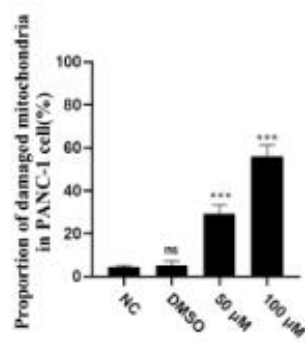

$\mathbf{J}$

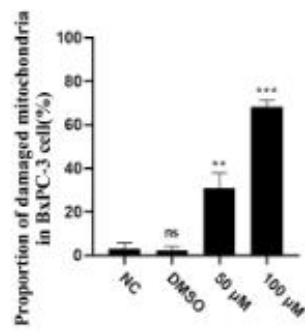

Figure 6. Compound 42 induced ferroptosis in pancreatic cancer cells. A-C: Western blot analysis of GPX4 expression in PANC-1 (A), BxPC-3 (B) and HepG-2 (C) cells treated with different concentration of Compound 42. D-E: Western blot analysis of GPX4 expression in PANC-1 (D) and BxPC-3 (E) with different treatment time using $100 \mu \mathrm{M}$ of Compound 42. F: 
Rescue assay of expression of GPX4 caused by Compound 42 treatment in PANC-1 cells. G-J:

Ultrastructural analysis of mitochondria in PANC-1 (G) and BxPC-3 (I) cells after Compound 42 treatment. Red arrows indicated the ruptured outer membrane (scale bars: $1 \mu \mathrm{m}$ and 400 $\mathrm{nm}$ ), $\mathrm{H}$ and $\mathrm{J}$ represented the proportion of morphological changes of mitochondria in PANC-1 and BxPC-3 cells, respectively. ns: no significant difference; *: $P<0.05 ; * *: P<$ $0.01 ; * * *: P<0.001$. 


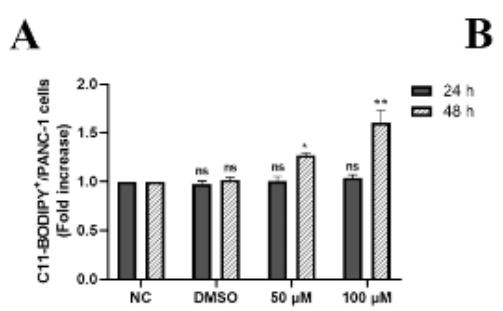

D

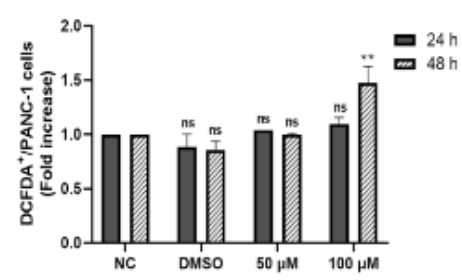

$\mathbf{F}$

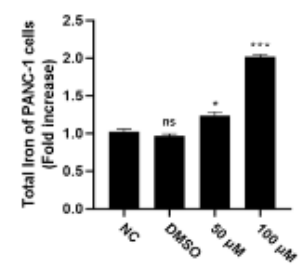

$\mathbf{H}$

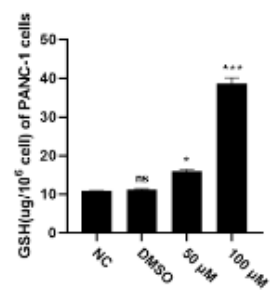

I

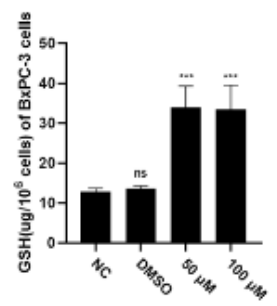

B

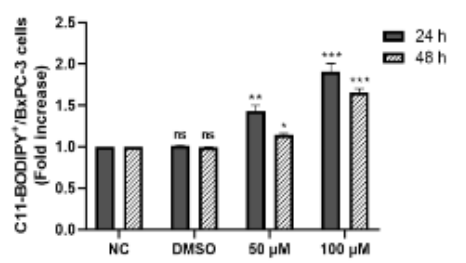

$\mathbf{E}$

C
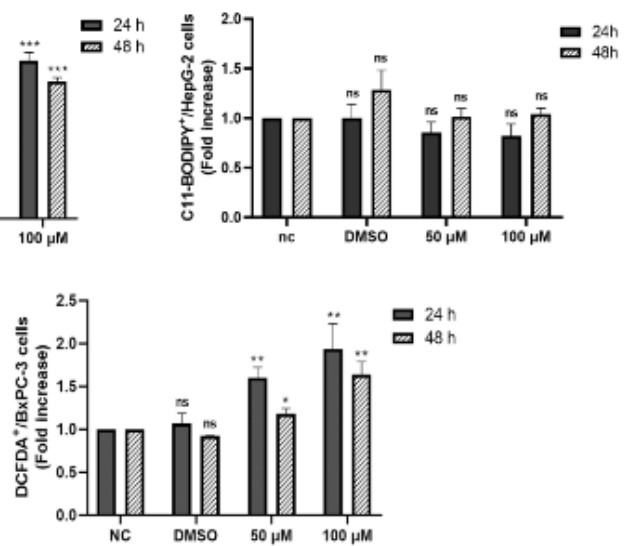

G
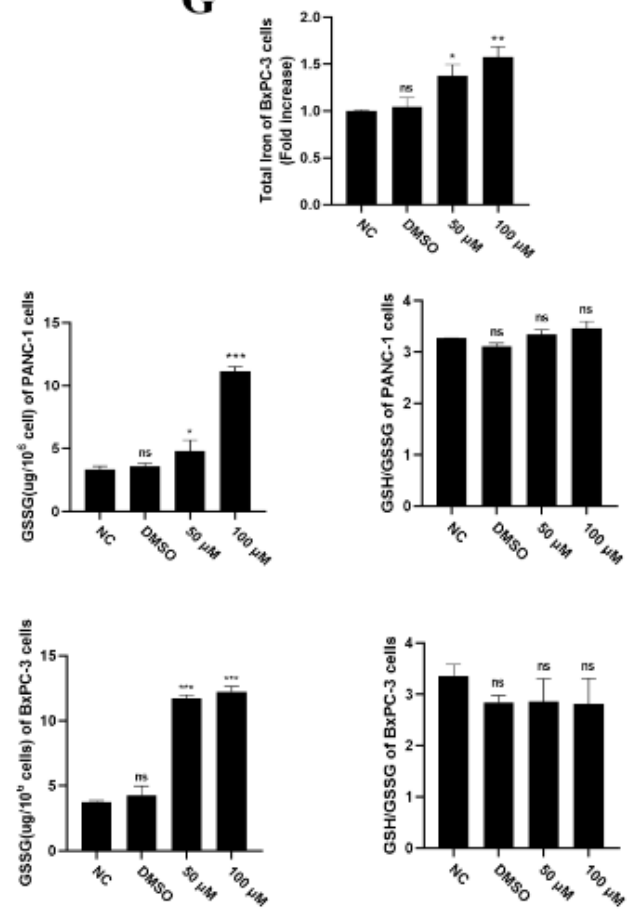

Figure 7. Compound 42 increased the concentration of lipid ROS and iron ions in

pancreatic cancer cells. A-C: Lipid ROS were accumulated by Compound 42 treatment in PANC-1 (A) and BxPC-3 (B) cells, but no obvious accumulation was detected in HepG-2 (C) cells. D-E: The intracellular total ROS was up-regulated by Compound 42 treatment in PANC-1 (D) and BxPC-3 (E) cells. F-G: The intracellular ferrous iron $\left(\mathrm{Fe}^{2+}\right)$ was accumulated by Compound 42 treatment in PANC-1 (F) and BxPC-3 (G) cells. H-I: 
Intracellular GSH and GSSG in PANC-1 (H) and BxPC-3 (I) cells treated with Compound 42 for $48 \mathrm{~h}$, and there were no obvious differences in GSH/GSSG between four groups. ns: no significant difference; *: $P<0.05 ; * *: P<0.01 ; * * *: P<0.001$. 


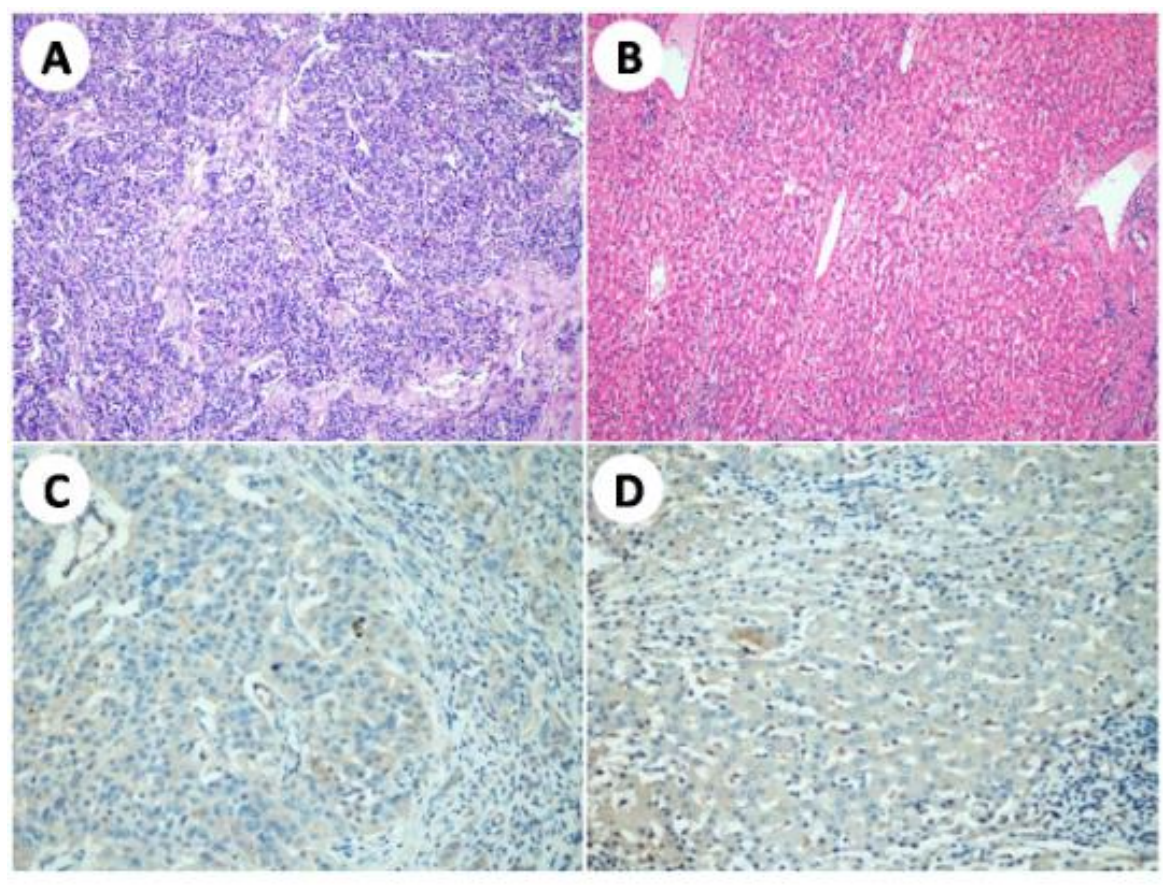

Supplementary Figure 1. No expression of KLK7 in both liver cancer tissues and

adjacent liver tissues. A-B: HE staining of liver cancer tissues (A) and adjacent liver tissues

(B). (100x) C-D: IHC of KLK7 protein in liver cancer tissues (C) and adjacent liver tissues

(D) showed that no expression of KLKL7 was observed in liver cancer and adjacent liver tissues $(200 \times)$. 
A

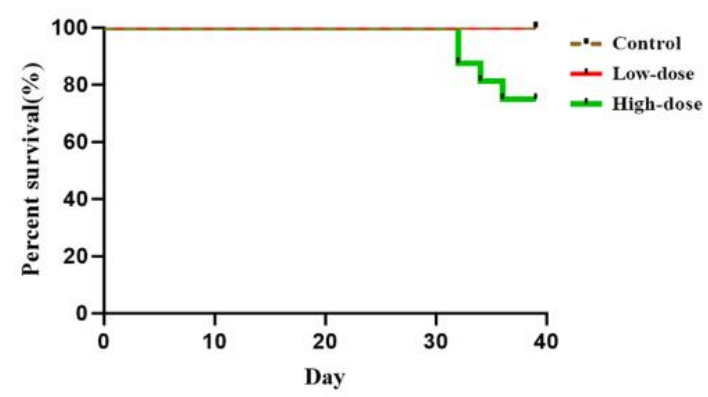

B

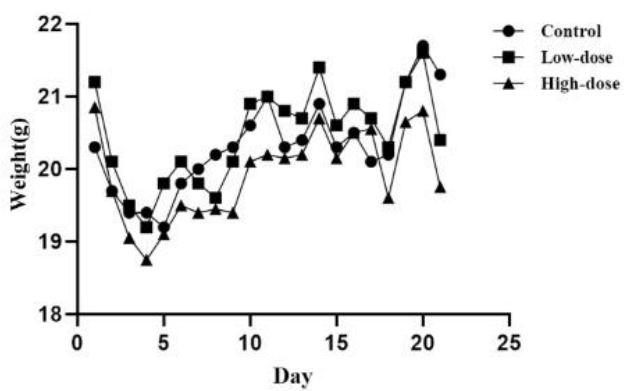

Spleen

Lung

Kidney

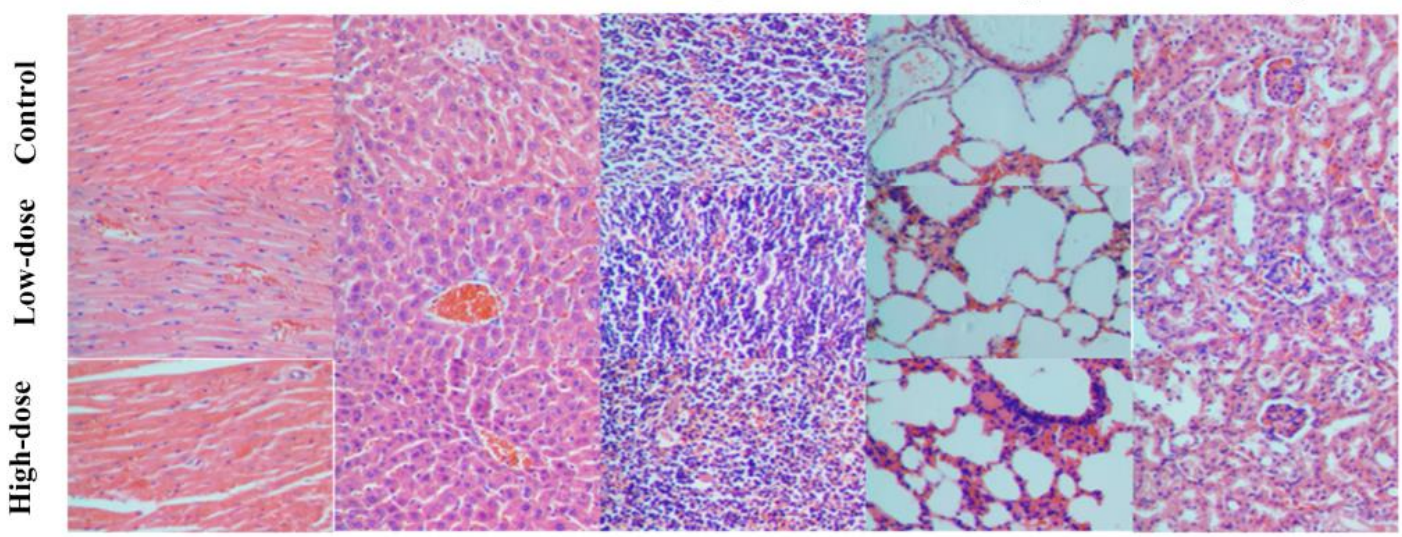

Supplementary Figure 2. Compound 42 had no obvious toxicity in vivo. A: High dose of

Compound $42(85 \mu \mathrm{g} / \mathrm{g})$ had little influence on survival rate of mice $(\mathrm{n}=16)$. B: The mice injected with high dose and low dose $(17 \mu \mathrm{g} / \mathrm{g})$ of Compound 42 had no obvious weight difference compared to control group (DMSO-only). C: After a 39-day observation, the tissues of heart, liver, spleen, lung and kidney of mice had no obvious lesions (200x). 


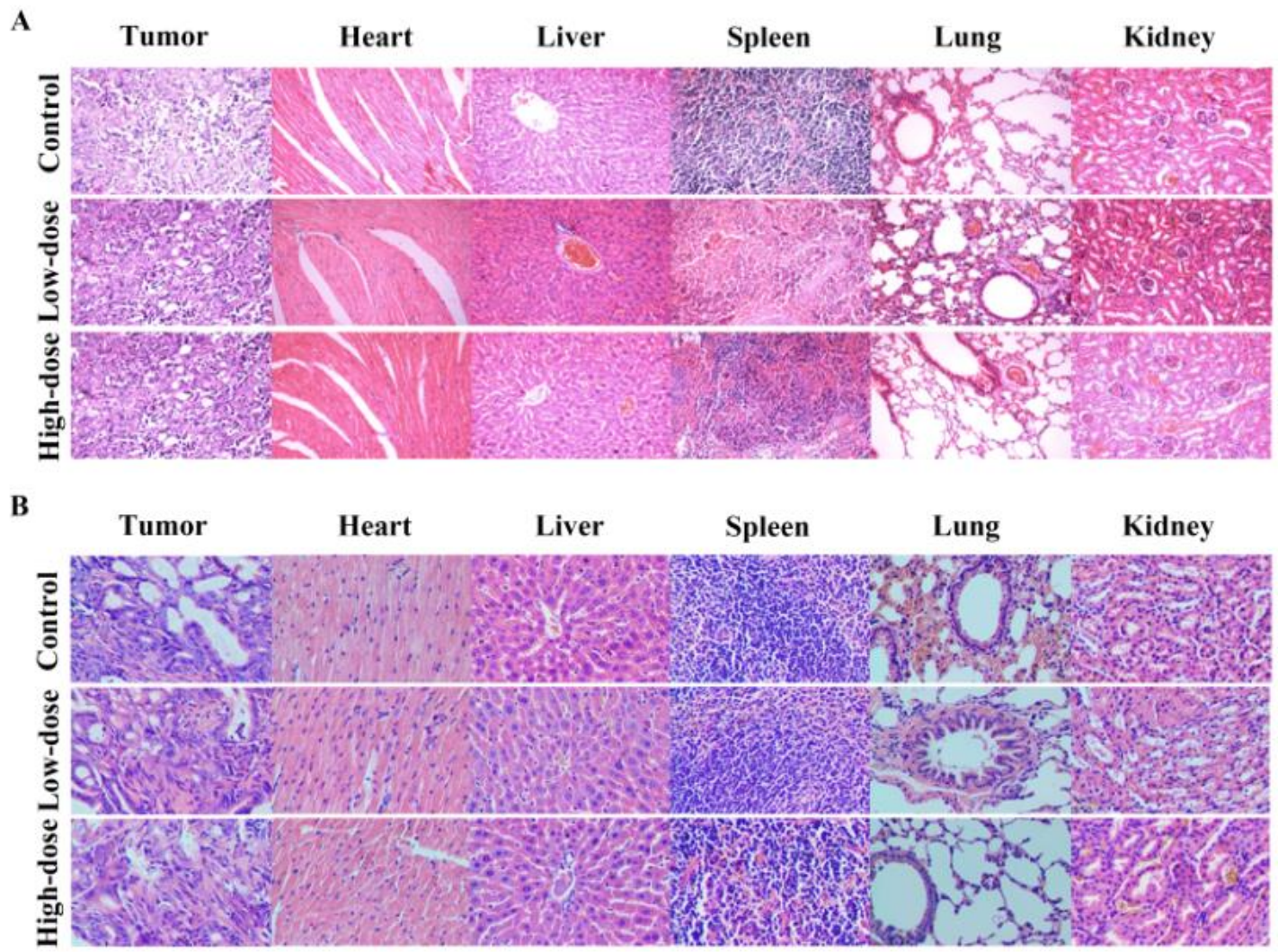

\section{Supplementary Figure 3. Compound 42 had no obvious toxicity in CDX and PDX mouse}

model. A-B: The tumor and other tissues of mouse in each group were sectioned, the H\&E

staining was performed to observe the morphological characteristics of the tissues (200x) in

CDX (A) and PDX (B) mouse model. 
mAU

A

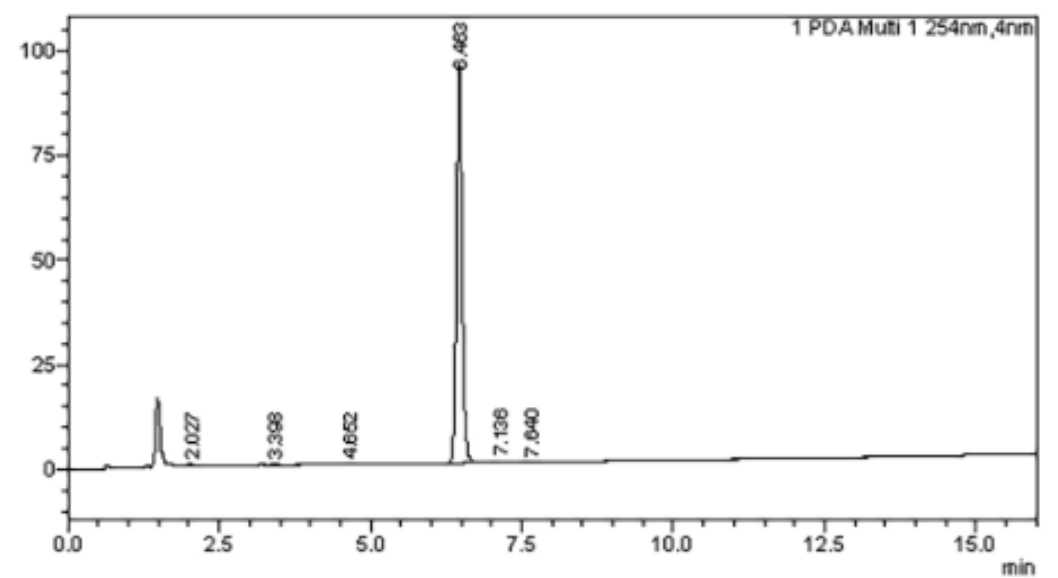

B
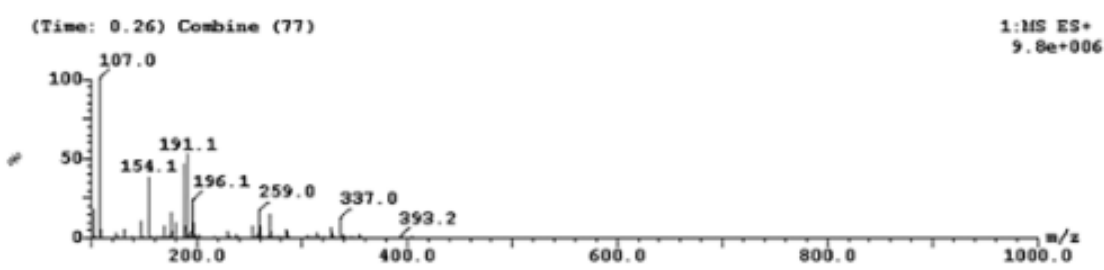

(Time: 1.40) Conbine (421)
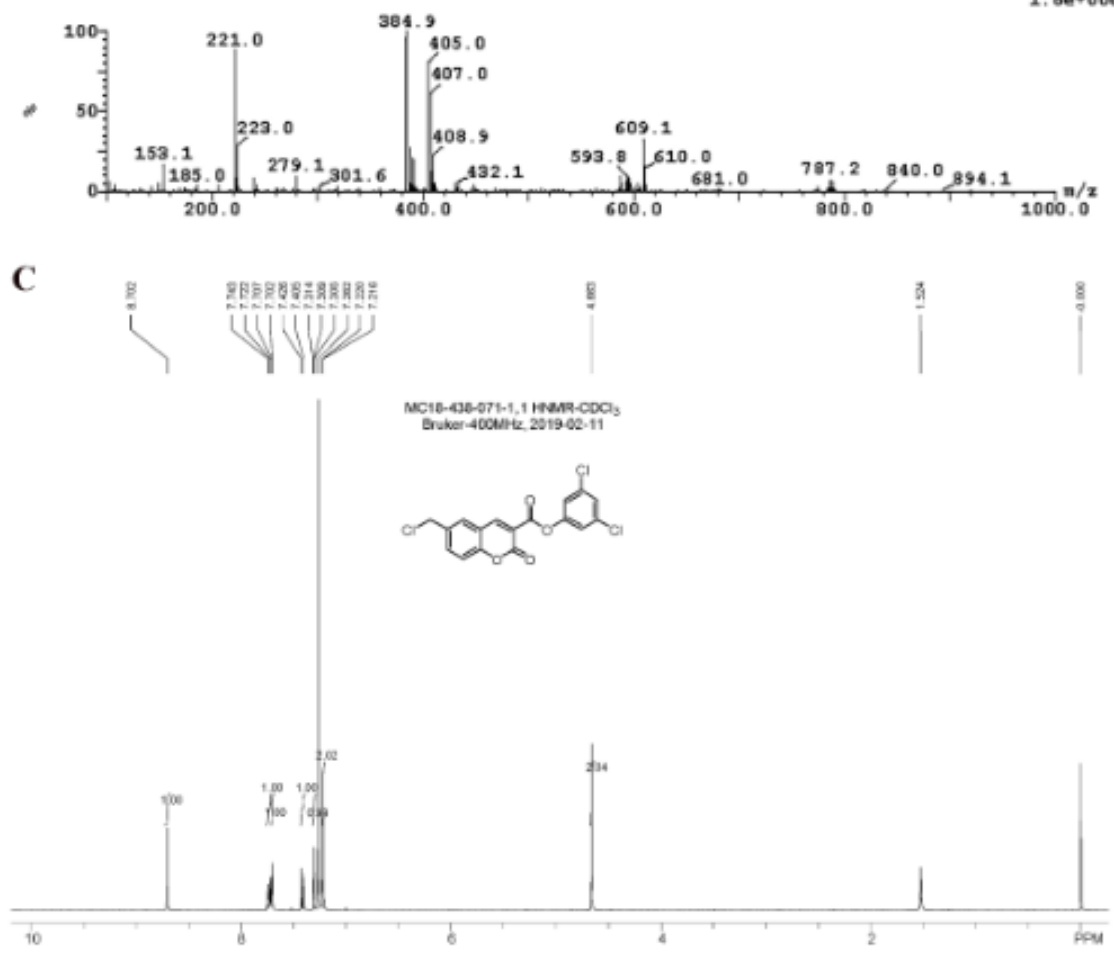

Supplementary Figure 4. Identification of Compound 42. A: Analysis of purification of

Compound 42 by HPLC. B: Analysis of Compound 42 by Mass spectrometry. C: Analysis of Compound 42 by nuclear magnetic resonance. 
$\mathbf{A}$

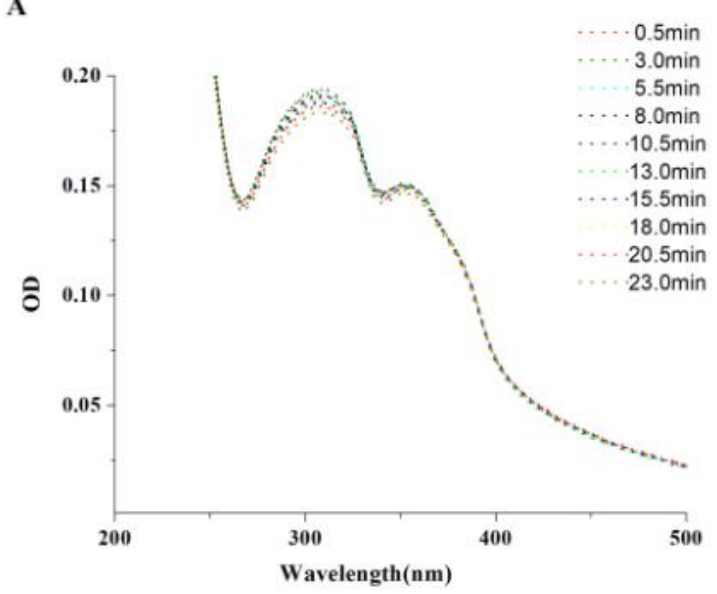

C

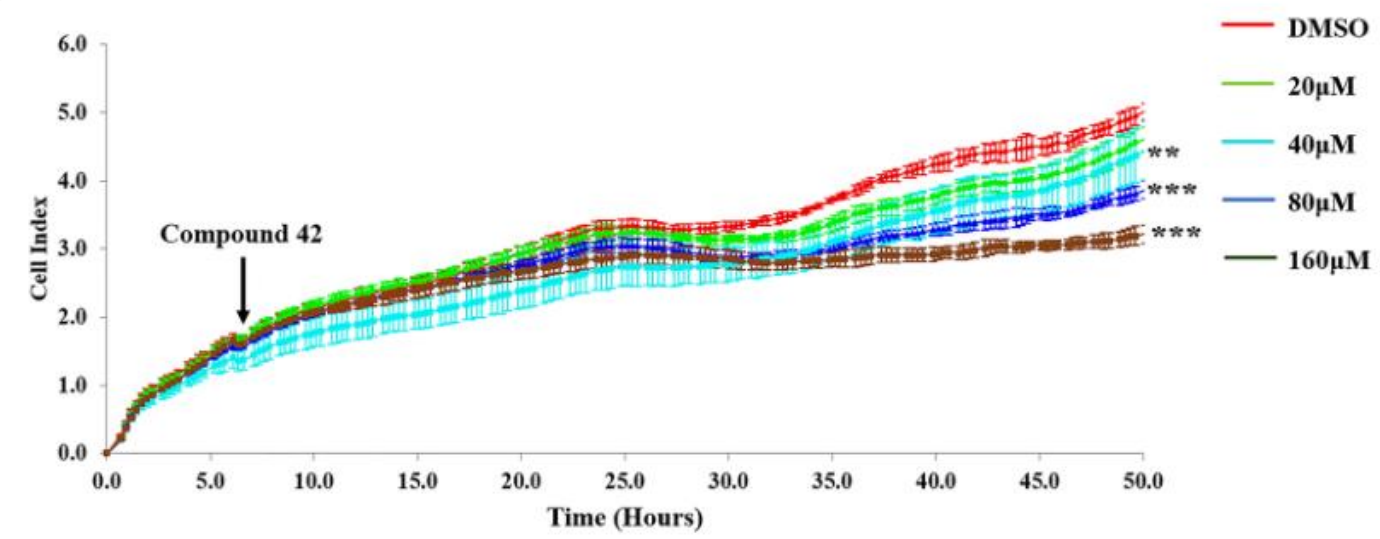

Supplementary Figure 5. The stability and inhibitory ability of compound 42. A: The absorption spectra of compound 42 at $20 \mu \mathrm{M}$ was measured every $2.5 \mathrm{sec}$ from $0.5 \mathrm{sec}$ to 23 min in PBS solution ( $\mathrm{pH}=7.4)$ using Ultraviolet spectrophotometer. B: The degradation of compound 42 in PBS ( $\mathrm{pH}=7.4)$ solution was analyzed during $30000 \mathrm{sec}$ at $305 \mathrm{~nm}$ by Ultraviolet spectrophotometry. C: The inhibitory ability of different concentration of Compound 42 pre-incubated with cell culture medium in PANC-1 cell was evaluated by RTCA during 50 h. **: $P<0.01 ; * * *: P<0.001$. 